\title{
Vertical dispersion of Lagrangian tracers in fully developed stably stratified turbulence
}

\author{
N.E. Sujovolsky and P.D. Mininni \\ Universidad de Buenos Aires, Facultad de Ciencias Exactas y Naturales, Departamento de Física, \\ \& IFIBA, CONICET, Ciudad Universitaria, Buenos Aires 1428, Argentina.
}

\begin{abstract}
We study the effect of different forcing functions and of the local gradient Richardson number $\mathrm{Ri}_{\mathrm{g}}$ on the vertical mixing of Lagrangian tracers in stably stratified turbulence under the Boussinesq approximation, and present a wave and continuous-time random walk model for single- and twoparticle vertical dispersion. The model consists of a random superposition of linear waves with their amplitude based on the observed Lagrangian spectrum of vertical velocity, and a random walk process to capture overturning that depends on the statistics of $\mathrm{Ri}_{\mathrm{g}}$ among other Eulerian quantities. The model is in good agreement with direct numerical simulations of stratified turbulence, where single- and two-particle dispersion differs from the homogeneous and isotropic case. Moreover, the model gives insight into the mixture of linear and non-linear physics in the problem, as well as on the different processes responsible for vertical turbulent dispersion.
\end{abstract}

\section{INTRODUCTION}

Stably stratified turbulence (SST) is common in geophysical flows, as the ocean and the atmosphere are usually in a turbulent state and affected by stratification (and rotation at the largest scales), making it of fundamental importance in the study of dispersion of pollutants, transport of nutrients, and turbulent mixing in a wide range of scales [1-4. As stably stratified turbulence is anisotropic, it is also inherently different from homogeneous isotropic turbulence (HIT) [5 8 . In SST, the stratification suppresses the vertical velocity, confining the flow into a quasi-horizontal layered motion, and generating vertically sheared horizontal winds (VSHWs) with high vertical variability 9. The stratification also results in a restoring force, allowing for the excitation of waves that can coexist with the turbulence.

As a result, vertical and horizontal turbulent transports in SST are fundamentally different. It has been speculated that horizontal mixing could be more efficient than in HIT due to the presence of VSHWs 9, 10. Indeed, horizontal dispersion is dominated by the VSHWs, as shown in direct numerical simulations and by our recent model for horizontal particle dispersion in [11. For vertical mixing and dispersion, stratification has some obvious and some not-so-obvious implications [12 14. On the one hand, the vertical velocity in SST is intermittent, implying that arguments based solely on mean values of the vertical velocity or its power spectrum could be misleading due to the spontaneous occurrence of extreme values [15, 16]. On the other hand, while it is well understood that in stratified turbulence as the stratification is increased the mean vertical velocity is quenched, vertical gradients also increase with increasing stratification, possibly balancing the vertical transport [5, 17, 18.

Mixing in stratified turbulence has been largely studied from an Eulerian point of view [19 24], but Lagrangian measurements with floaters are also common in the present, specially in oceanic measurements of waves and turbulence 2, where they are relevant to understand the transport of nutrients with applications for the fishing industry. Vertical dispersion is also important in the atmosphere [25, and particle dispersion has also been studied recently in atmospheric flows for forecasting purposes using Lagrangian models [26. In spite of this, there are few studies of stratified turbulence from the Lagrangian point of view [27]29], where linear theories of SST predict the bounding of particles in the vertical direction and the saturation for long times of single- and two-particle vertical dispersion [28. However, linear models cannot capture the effect of overturning, or of thermal diffusion, that can be relevant at intermediate times and dominate the dynamics of the vertical mixing at very long times [29. It is also worth noting that inertial particles with density different from that of the fluid are also relevant to study mixing, and have received substantial attention in HIT (see, e.g., [30, 31]), with a special emphasis on the mechanisms leading to its spatial distribution and clustering. However, mixing and distribution of inertial particles in SST have only been studied recently [4, 32, 33].

In this paper we present several direct numerical simulations (DNSs) of the Boussinesq equations with Reynolds buoyancy numbers $\mathrm{Rb}>1$ in two different domains: one isotropic (cubic), and the other anisotropic (an elongated domain with the horizontal sides longer than the vertical), and using two different mechanical forcing functions. We applied a random forcing (RND), and a Taylor-Green forcing (TG) which generates a coherent large-scale flow at the largest available scales, thus affecting vertical mixing. The Boussinesq Eulerian flow is evolved together with Lagrangian particles. We study single- and two-particle vertical dispersion, and analyze the role of the Froude number, the vertical shear, the large-scale flow, and the local gradient Richardson number in the vertical dispersion of particles. We also present a model for the vertical single- and two-particle vertical dispersion that is in good agreement with the DNS results. The model consist of a continuous-time random walk (CTRW) based on a previous model for HIT [34, 35] (to model trapping of tracers by turbulent eddies, and the effect of local overturning instabilities), and a 
random superposition of waves, and can capture the vertical dispersion of particles at all times and in all SST regimes considered. The superposition of linear and turbulent effects in the model allows us to identify the leading physical effects resulting in vertical dispersion at early and at late times in the flow (compared with the period of the internal gravity waves). Moreover, as all parameters in the model can be obtained from large-scale Eulerian data, the model could be used autonomously to obtain statistical predictions of particle dispersion provided a large-scale flow.

\section{NUMERICAL SIMULATIONS}

For this study we solved numerically the incompressible Boussinesq equations for the velocity $\mathbf{u}$ and for buoyancy (or "temperature") fluctuations $\theta$,

$$
\begin{aligned}
\partial_{t} \mathbf{u}+\mathbf{u} \cdot \nabla \mathbf{u} & =-\nabla p-N \theta \hat{z}+\nu \nabla^{2} \mathbf{u}+\mathbf{f} \\
\partial_{t} \theta+\mathbf{u} \cdot \nabla \theta & =N \mathbf{u} \cdot \hat{z}+\kappa \nabla^{2} \theta \\
\nabla \cdot \mathbf{u} & =0
\end{aligned}
$$

where $p$ is the correction to the hydrostatic pressure, $\nu$ is the kinematic viscosity, $\mathbf{f}$ is an external mechanical forcing, $N$ is the Brunt-Väisälä frequency (which sets the stratification), and $\kappa$ is the diffusivity. In terms of the density fluctuations $\rho$, the Brunt-Väisälä frequency is $N^{2}=-\left(g / \rho_{0}\right)(d \bar{\rho} / d z)$, with $d \bar{\rho} / d z$ the imposed (linear) background density stratification, and $\rho_{0}$ the mean density. We write the buoyancy field $\theta$ in units of velocity by defining $\theta=g \rho /\left(\rho_{0} N\right)$. All quantities are then made dimensionless using a characteristic length $L_{0}$ and a characteristic velocity $U_{0}$. All runs in this paper have a Prandtl number $\operatorname{Pr}=\nu / \kappa=1$.

The Boussinesq equations were solved in a three-dimensional periodic domain, using a parallelized and fully dealiased pseudospectral method, and a second-order Runge-Kutta scheme for time integration [36. In the turbulent steady state of each simulation we also injected $\mathcal{O}\left(10^{6}\right)$ Lagrangian particles, and integrated their trajectories in time using

$$
\mathbf{v}_{i}=\frac{d \mathbf{x}_{i}}{d t}=\mathbf{u}\left(\mathbf{x}_{i}, t\right)
$$

where the subindex $i$ labels each particle. Here and in the following, the velocity of Lagrangian particles and its Cartesian components are represented as $\mathbf{v}=\left(v_{x}, v_{y}, v_{z}\right)$, while the Eulerian fluid velocity is given by $\mathbf{u}=\left(u_{x}, u_{y}, u_{z}\right)$. Integration of particles' trajectories was done using a second-order Runge-Kutta method in time, and a three-dimensional cubic spline interpolation to estimate Lagrangian velocities at the particles positions $\mathbf{x}_{i}$ from the velocity $\mathbf{u}$ in the regular Eulerian grid [37. All simulations were done using the GHOST code (Geophysical High-Order Suite for Turbulence), recently extended to work with non-cubic boxes 38 .

Equations (1) and (2) have two controlling dimensionless parameters, the Reynolds and the Froude numbers, respectively given by

$$
\operatorname{Re}=\frac{L U}{\nu}, \quad \operatorname{Fr}=\frac{U}{L N},
$$

where $L$ and $U$ are respectively the characteristic Eulerian integral length and r.m.s. velocity of the flow. From Eq. (5) we can also define the buoyancy Reynolds number

$$
\mathrm{Rb}=\mathrm{ReFr}^{2}
$$

which gives an estimation of how turbulent the flow is at the buoyancy scale $L_{b}=U / N$, and as a result can be expected to play an important role in turbulent mixing. In the following we will consider simulations with $\mathrm{Rb}>1$. The Ozmidov scale, $L_{o z}=2 \pi / k_{o z}$ (with $k_{o z}=\sqrt{N^{3} / \epsilon}$ ), will also play an important role in the following discussions, as for scales sufficiently small when compared with $L_{o z}$, the flow is expected to recover isotropy. When Rb $>1$ the Ozmidov scale is larger than the Kolmogorov dissipation scale $\eta$, and quasi-isotropic turbulent mixing can thus be expected to take place at small scales. Another parameter that will be useful to quantify small scale turbulence and mixing is the local gradient Richardson number

$$
\mathrm{Ri}_{g}=\frac{N\left(N-\partial_{z} \theta\right)}{\left(\partial_{z} u_{\perp}\right)^{2}},
$$

where $u_{\perp}$ is the horizontal velocity. When $\mathrm{Ri}_{g}<1 / 4$ the flow can develop shear instabilities 39 , while for $\mathrm{Ri}_{g}<0$ local overturning can take place. 
Other relevant parameters for the next sections are the Eulerian turnover time at the Ozmidov scale $\tau_{o z}=L_{o z} / U_{z}$ (with $U_{z}$ the characteristic Eulerian vertical velocity), and the energy-containing (or integral) isotropic and parallel length scales

$$
\begin{gathered}
L=2 \pi \frac{\int E_{V}(k) k^{-1} d k}{\int E_{V}(k) d k}, \\
L_{\|}=2 \pi \frac{\int E_{V}\left(k_{\|}\right) k_{\|}^{-1} d k_{\|}}{\int E_{V}(k) d k},
\end{gathered}
$$

where $E_{V}(k)$ and $E_{V}\left(k_{\|}\right)$are respectively the isotropic and parallel kinetic energy spectra. From these lengths we can also define an energy-containing isotropic wavenumber as $K=2 \pi / L$, and an energy-containing parallel wavenumber as $K_{\|}=2 \pi / L_{\|}$.

The numerical simulations were performed in three-dimensional periodic domains with different aspect ratios. A first set of runs has an isotropic box with domain lengths $L_{x}=L_{y}=L_{z}$ (equal to $2 \pi$ in dimensionless units) and isotropic linear resolution $n_{x}=n_{y}=n_{z}$, and therefore with an aspect ratio of the vertical to horizontal lengths of 1:1. Another set of simulations was done in elongated boxes with sizes $L_{x}=L_{y}=\alpha L_{z}$ and resolution $n_{x}=n_{y}=\alpha n_{z}$. Thus, the aspect ratio of the domain is $1: \alpha$, and we will consider in the following $\alpha=4$ or 8 . Note that in all cases the spatial resolution is isotropic, i.e., the distance between grid points is the same in the three directions, $\Delta_{x}=\Delta_{y}=\Delta_{z}$, and thus isotropy can in principle be recovered by the flow at the smallest scales.

In each domain, simulations were done using two different forcing functions. Some simulations were forced with Taylor-Green (TG) forcing (see, e.g., [10, 38, 40]), which only excites directly the two horizontal components of the velocity field, and has vertical shear. The geometry of the large-scale flow generated by this forcing is that of pairs of counter-rotating horizontal vortices at large scales, and the expression of the forcing is

$$
\mathbf{f}_{\mathbf{T G}}=f_{0}(\sin (x) \cos (y) \cos (\alpha z),-\cos (x) \sin (y) \cos (\alpha z), 0)
$$

The effectively forced wavenumber is then $k_{f}=\left(2+\alpha^{2}\right)^{1 / 2}$. Note that changing the aspect ratio of the domain modifies the factor $\alpha=L_{x} / L_{z}$ and thus, the strength of vertical gradients in the flow. For $\alpha=1$ (isotropic domain) $k_{f} \approx 1.7$, while for $\alpha=4$ or 8 we obtain respectively $k_{f} \approx 4.2$ or 8.1 . The flow generated by these forces (for $\alpha \neq 1$ ) still has a large-scale circulation at $k_{x}=k_{y}=1$, while developing stronger shear in the vertical direction as $\alpha$ is increased (see [38] for more details).

Other simulations were done using a random isotropic three-dimensional forcing (RND), with a correlation time $\tau_{c o r r}$ of half an eddy turn-over time. A forcing with random phases in the Fourier shell $k_{f}=\alpha$ is computed at a given time as

$$
\mathbf{f}_{\mathbf{1}}=f_{0} \sum_{|\mathbf{k}| \in\left[k_{f}, k_{f}+1\right)} \Re\left[i \mathbf{k} \times \hat{\mathbf{u}}_{\mathbf{k}} e^{i\left(\mathbf{k} \cdot \mathbf{r}+\varphi_{\mathbf{k}}\right)}\right]
$$

where $\Re$ stands for the real part, $\hat{\mathbf{u}}_{\mathbf{k}}$ is a unit vector, and $\varphi_{\mathbf{k}}$ are uniformly distributed random phases. The actual forcing $\mathbf{f}_{\mathbf{R N D}}$ is obtained by slowly interpolating the forcing from a previous random state $\mathbf{f}_{\mathbf{0}}$ to the new random state $\mathbf{f}_{\mathbf{1}}$, in such a way that $\mathbf{f}_{\mathbf{R N D}}=\mathbf{f}_{\mathbf{1}}$ after $\tau_{\text {corr }}$. The process is then repeated to obtain a slowly evolving random forcing. As the forcing wavenumber depends on the aspect ratio, in the isotropic box $k_{f}=1$ while in the elongated domains $k_{f}=4$ or 8 , similarly to the Taylor-Green case. However, note that in this case the choice $k_{f}=\alpha$ to maintain the forcing isotropic for all aspect ratios also implies that, as the aspect ratio $1: \alpha$ is decreased and the forcing is applied (isotropically) at smaller scales, the Reynolds number (based on the energy containing scale) will also decrease.

All flows were evolved from $\mathbf{u}=\theta=0$, and once they reached the turbulent steady state Lagrangian particles were injected and integrated in time together with the flow. The list of all runs with their respective relevant parameters is presented in table I. Runs are labeled using the forcing (TG or RND), a subindex for the inverse aspect ratio $(\alpha=1$, 4 or 8$)$, and a number indicating the value of the Brunt-Väisälä frequency $(N=4,8$ or 12$)$. As mentioned above, note that run $\mathrm{RND}_{4} 8$ has a lower Re than, e.g., run $\mathrm{TG}_{4} 8$ (although it has the same spatial resolution and kinematic viscosity), as the isotropic forcing at $k_{f}=\alpha=4$ in the $\mathrm{RND}_{4} 8$ run results in a smaller integral length scale when compared to the $\mathrm{TG}_{4} 8$ run, which has a large-scale flow at horizontal scales (with $k_{\perp} \approx 1$ ) with shear at smaller vertical scales (with $k_{\|}=4$ ). However, note run $\mathrm{RND}_{4} 8$ also has a larger Fr, thus resulting in a larger Rb. To explore the effect of varying Re and $\mathrm{Rb}$, while keeping the forcing and aspect ratio fixed and Fr approximately the same, runs $\mathrm{RND}_{4} 8$ and $\mathrm{RND}_{4} 8 \mathrm{~B}$ to $\mathrm{RND}_{4} 8 \mathrm{D}$ were done at decreasing spatial resolution and at increasing values of $\nu=\kappa$. 


\begin{tabular}{|c|c|c|c|c|c|c|c|c|c|c|c|c|}
\hline Run & Aspect ratio & $n_{x}=n_{y}$ & $n_{z}$ & Forcing & $N$ & $R e$ & $F r$ & $R_{b}$ & $R$ & $2 \pi / N$ & $L_{o z}$ & $\tau_{o z}$ \\
\hline $\mathrm{TG}_{1} 4$ & $1: 1$ & 512 & 512 & TG & 4 & 7000 & 0.04 & 11 & 0.12 & 1.57 & 0.28 & 1.2 \\
\hline $\mathrm{TG}_{1} 8$ & $1: 1$ & 512 & 512 & TG & 8 & 8000 & 0.02 & 3 & 0.03 & 0.79 & 0.1 & 0.8 \\
\hline $\mathrm{TG}_{4} 4$ & $1: 4$ & 768 & 192 & TG & 4 & 10000 & 0.05 & 25 & 0.25 & 1.57 & 0.36 & 1.4 \\
\hline $\mathrm{TG}_{4} 8$ & $1: 4$ & 768 & 192 & TG & 8 & 14000 & 0.03 & 13 & 0.09 & 0.79 & 0.14 & 0.7 \\
\hline $\mathrm{TG}_{4} 12$ & $1: 4$ & 768 & 192 & TG & 12 & 15000 & 0.02 & 4 & 0.03 & 0.52 & 0.07 & 1.0 \\
\hline $\mathrm{TG}_{8} 8$ & $1: 8$ & 2048 & 256 & $\mathrm{TG}$ & 8 & 35000 & 0.03 & 30 & 0.30 & 0.79 & 0.18 & 0.8 \\
\hline $\mathrm{RND}_{1} 4$ & $1: 1$ & 512 & 512 & RND & 4 & 6000 & 0.07 & 29 & 0.06 & 1.57 & 0.24 & 0.9 \\
\hline $\mathrm{RND}_{1} 8$ & $1: 1$ & 512 & 512 & RND & 8 & 8000 & 0.03 & 7 & 0.02 & 0.79 & 0.07 & 0.3 \\
\hline $\mathrm{RND}_{4} 8$ & $1: 4$ & 768 & 192 & RND & 8 & 3000 & 0.11 & 36 & 0.16 & 0.79 & 0.17 & 0.7 \\
\hline $\mathrm{RND}_{4} 8 \mathrm{~B}$ & $1: 4$ & 512 & 128 & RND & 8 & 2000 & 0.10 & 20 & 0.07 & 0.79 & 0.14 & 0.6 \\
\hline $\mathrm{RND}_{4} 8 \mathrm{C}$ & $1: 4$ & 256 & 64 & RND & 8 & 800 & 0.17 & 23 & 0.02 & 0.79 & 0.16 & 0.8 \\
\hline $\mathrm{RND}_{4} 8 \mathrm{D}$ & $1: 4$ & 128 & 32 & RND & 8 & 300 & 0.20 & 12 & 0.25 & 0.79 & 0.15 & 0.2 \\
\hline
\end{tabular}

TABLE I: Relevant parameters of the simulations. The aspect ratio gives the vertical to horizontal aspect ratio of the spatial domain $1: \alpha, n_{x}, n_{y}$, and $n_{z}$ are the grid points in each spatial direction, forcing indicates the forcing function, Re is the Reynolds number, Fr is the Froude number, $\mathrm{Rb}$ is the buoyancy Reynolds number, $R$ is the fraction of particles with $\mathrm{Ri}_{\mathrm{g}}<0$, $L_{o z}$ is the Ozmidov length scale, and $\tau_{o z}=L_{o z} / U_{z}$ is the Eulerian turnover time at the Ozmidov scale.
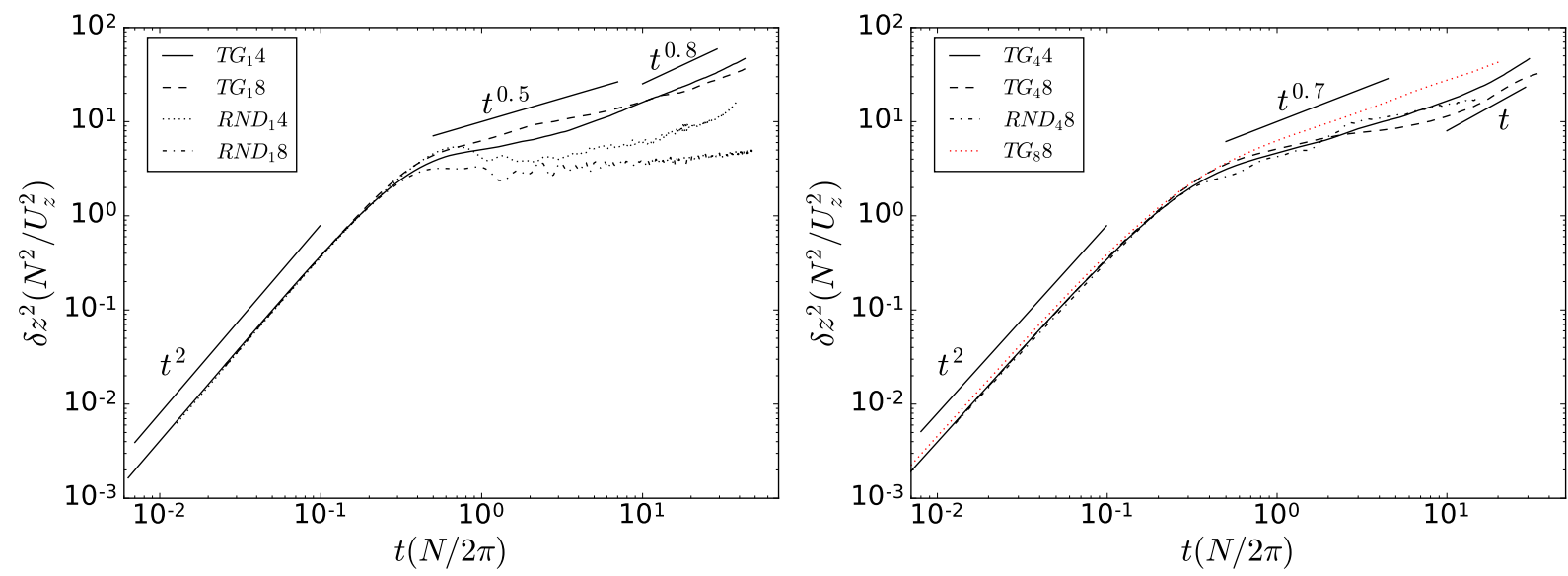

FIG. 1: Mean vertical dispersion $\delta z^{2}$ for (left) runs in isotropic domains (boxes with aspect ratio 1:1), and (right) runs in elongated domains with aspect ratio 1:4, in both figures with TG and RND forcing, and different Brunt-Väisälä frequencies. The dispersion is normalized by $U_{z}^{2} / N^{2}$, the ratio of the squared mean vertical velocity to the Brunt-Väisälä frequency, and time is normalized by the Brunt-Väisälä period. Power laws are indicated as references.

\section{SINGLE-PARTICLE VERTICAL DISPERSION IN STABLY STRATIFIED TURBULENCE}

Particle dispersion in SST is inherently different from HIT as stratification suppresses vertical dispersion. As mentioned in the Introduction, linear models of SST predict the saturation of the vertical dispersion for $t \approx 2 \pi / N$, as the displacement of particles is in practice vertically bounded by the stratification, resulting in an oscillatory motion of the particles 28. This is confirmed by numerical simulations at moderate buoyancy Reynolds number [5, 12 .

We computed the single-particle vertical dispersion as $\delta z^{2}=\left\langle\left[z_{i}(t)-z_{i}(0)\right]^{2}\right\rangle_{i}$, where $i$ is the particle label, and the average is computed over all particles. Figure 1 shows the resulting mean vertical dispersion in our simulations, for TG and RND forcing, different aspect ratios, and different Brunt-Väisälä frequencies (and thus, different Froude numbers). Time is normalized by $2 \pi / N$ (the Brunt-Väisälä period), while $\delta z^{2}$ is normalized by $\left(U_{z} / N\right)^{2}$ (following the normalization used in [29]). With this normalization all curves collapse from $t=0$ until $t \approx 2 \pi / N$, in a time range where they display ballistic behavior $\delta z^{2} \sim t^{2}$. This indicates that the early-time vertical dispersion is dominated by the waves, in good agreement with previous studies [11, 29]: particles are first displaced ballistically by the internal gravity waves, which for $\mathrm{Fr}<1$ are faster than the large-scale turbulent eddies. This behavior finishes after one wave period, resulting in a change in the growth of $\delta z^{2}(t)$. In some cases (see runs $\mathrm{RND}_{1} 4$ and $\mathrm{RND}_{1} 8$ in Fig. 1), $\delta z^{2}(t)$ grows very slowly or even saturates at late times, displaying a plateau. This saturation was reported before in simulations of SST at moderate Re and Rb numbers [11, 29], where a very slow growth at late times was attributed to the effect of molecular diffusion. However, some of our runs (all TG runs even at moderate Rb, and simulations with RND forcing at higher Rb in elongated domains) display a more efficient mixing (i.e., a faster growth of $\delta z^{2}(t)$ 

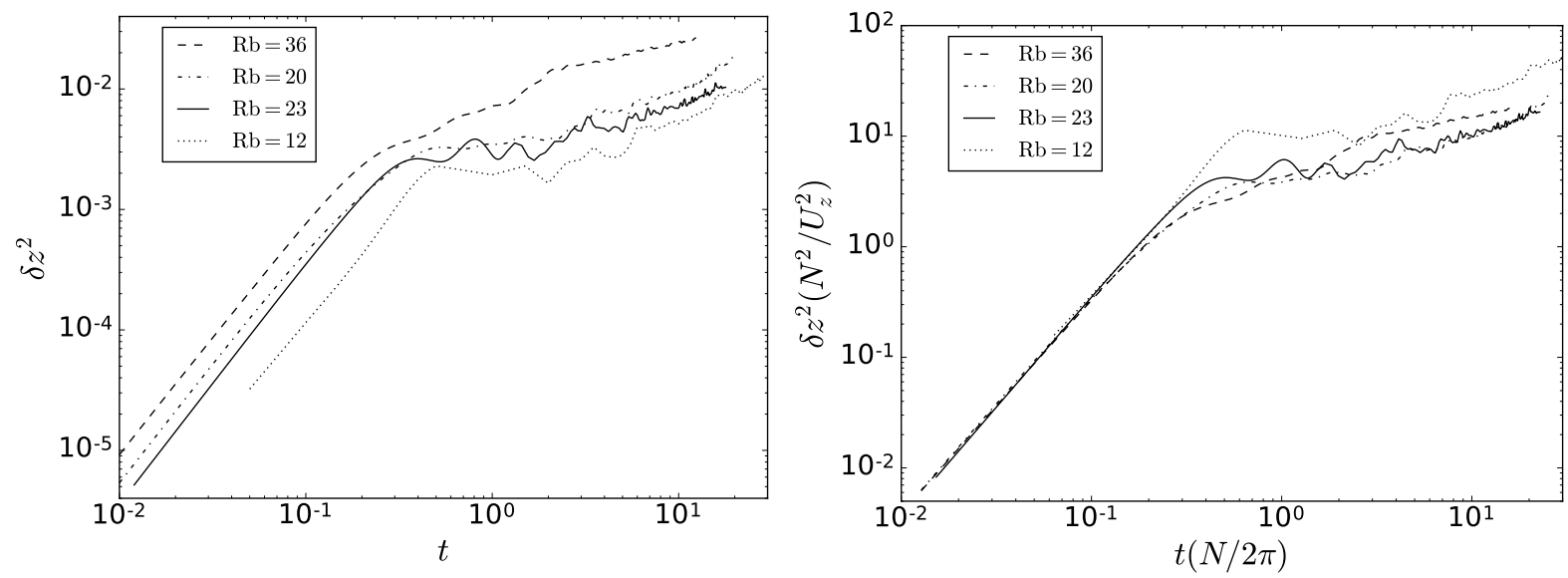

FIG. 2: Mean vertical dispersion $\delta z^{2}$ for RND runs in domains with aspect ratio $1: 4$ and with $N=8$ (run RND 8 and runs $\mathrm{RND}_{4} 8 \mathrm{~B}$ to $\mathrm{RND}_{4} 8 \mathrm{D}$, from higher to lower Re and Rb. Left: $\delta z^{2}(t)$ without normalization. Right: same, normalized by the ratio $U_{z}^{2} / N^{2}$, and with time normalized by the Brunt-Väisälä period.

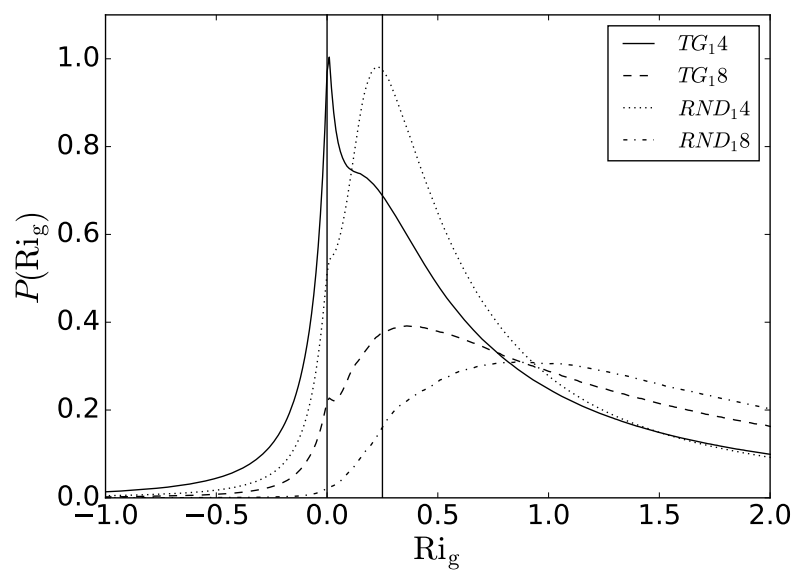

FIG. 3: PDFs of the Eulerial local gradient Richardson number $\mathrm{Ri}_{g}$, for all runs in isotropic domains (runs $\mathrm{TG}_{1} 4$ and $\mathrm{TG}_{1} 8$ with TG forcing and $N=4$ and 8 respectively, and runs $\mathrm{RND}_{1} 4$ and $\mathrm{RND}_{1} 8$ with RND forcing and $N=4$ and 8 respectively). Vertical solid lines at $\mathrm{Ri}_{g}=0$ and $1 / 4$ are shown as references.

for $t>2 \pi / N)$ when compared with the runs that display the plateau.

The enhanced mixing after $t>2 \pi / N$ seems to be controlled, at least for for RND forcing, by Rb, suggesting it may be the result of turbulence generated by shear instabilities or by overturning events. Figure 2 shows the single-particle vertical dispersion for several runs with RND forcing, and with the same parameters as $\mathrm{RND}_{4} 8$ ( $\mathrm{runs}^{\mathrm{RND}} \mathrm{R}_{4} 8 \mathrm{~B}$ to $\mathrm{RND}_{4} 8 \mathrm{D}$ ), but with different spatial resolution and values of $\mathrm{Rb}$ (by decreasing $\mathrm{Re}$ ). Run $\mathrm{RND}_{4} 8 \mathrm{D}$, with the lowest values of $\operatorname{Re} \approx 300$ and of $\mathrm{Rb} \approx 12$, displays a saturation in $\delta z^{2}(t)$ at $t N /(2 \pi) \approx 7 \times 10^{-1}$, a plateau until $t N /(2 \pi) \approx 5$, and then a slow growth. As Rb increases the plateau shortens, until it completely disappears for run $\mathrm{RND}_{4} 8$ (with $\mathrm{Re} \approx 3000$ and $\mathrm{Rb} \approx 36$ ).

The case of TG forcing is different, as the plateau in $\delta z^{2}(t)$ at intermediate times is not present even in runs at moderate $\mathrm{Rb}$. Although turbulence plays an important role in the mixing at high $\mathrm{Rb}$, the TG forcing function generates a coherent large-scale flow which creates strong fronts and helps instabilities to develop [38, enhancing vertical dispersion even at values of $\mathrm{Rb}$ which are low when compared to the RND case. In the next section we study the gradient Richardson number $\mathrm{Ri}_{g}$, with a special focus on the TG simulations, to characterize the features of this flow that result in differences in the vertical dispersion. 


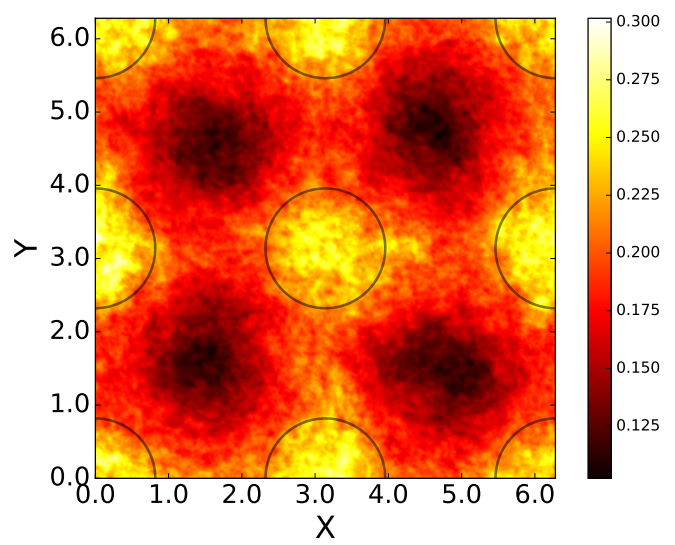

FIG. 4: (Color online). Vertically averaged absolute value of the Eulerian vertical velocity, $\left\langle\left|u_{z}\right|\right\rangle_{z}$, for run TG 4 (TG forcing, $N=4$, and aspect ratio 1:4). Bright regions correspond to large vertical velocities in absolute value. As the domain is periodic in both $x$ and $y$ directions, the regions with large $\left\langle\left|u_{z}\right|\right\rangle_{z}$ can be enclosed by four circles (cylinders when extended in the $z$ direction), indicated as a reference by the black solid lines.
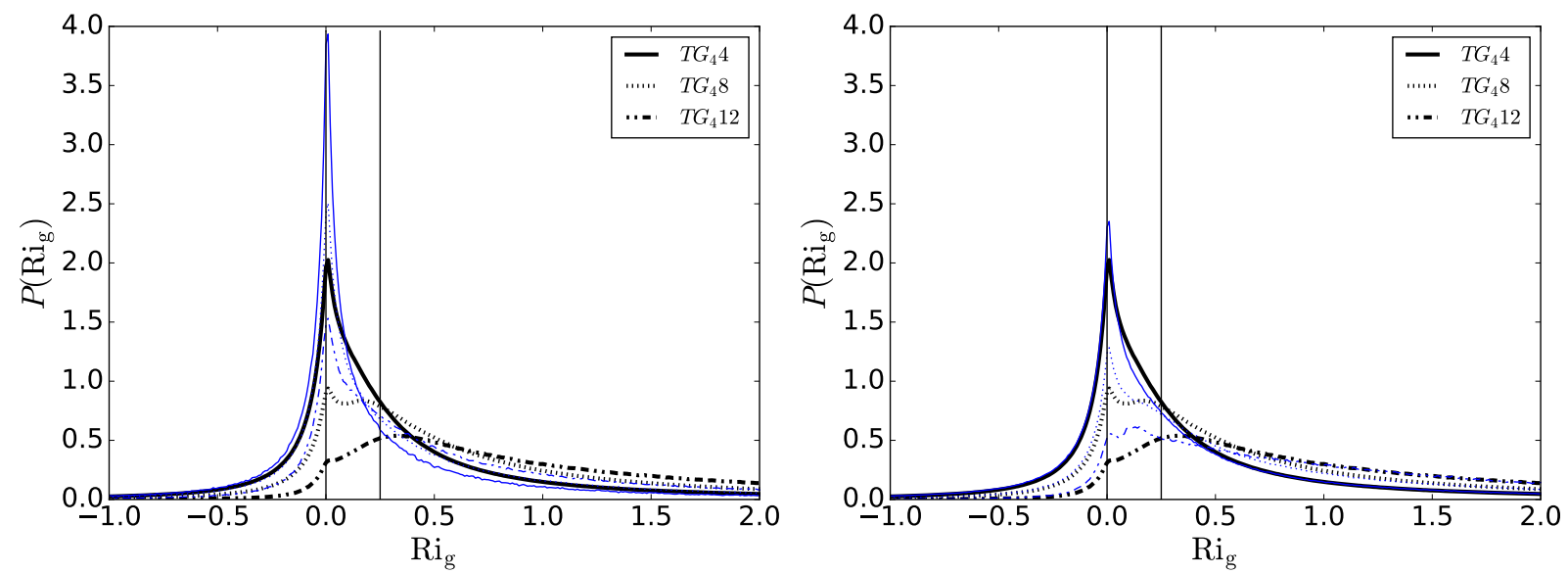

FIG. 5: (Color online). Both panels show, in black thick lines, the Lagrangian PDFs of the gradient Richardson number Ri ${ }_{g}$ for TG runs in boxes with 1:4 aspect ratio. This PDF is compared with (left) the PDFs of $\mathrm{Ri}_{g}$ restricted to Lagrangian velocities $\left|v_{z}\right|>\left\langle\left|v_{z}\right|\right\rangle+2 \sigma_{v_{z}}$ (where $\sigma_{v_{z}}$ is the dispersion in $v_{z}$ ) in thin (blue) lines, and (right) the $\mathrm{PDFs}$ of $\mathrm{Ri}_{g}$ restricted to particles in the circular regions indicated in Fig. 4. Vertical lines at $\mathrm{Ri}_{g}=0$ and $\mathrm{Ri}_{g}=1 / 4$ are shown as references.

\section{THE LOCAL GRADIENT RICHARDSON NUMBER}

\section{A. General properties of the local gradient Richardson number}

The local gradient Richardson number provides a measure of the vertical stability of stratified flows. When $\operatorname{Ri}_{g}(\mathbf{r})<$ $1 / 4$ pointwise, local shear instabilities can take place [41, while if $\operatorname{Ri}_{g}<0$, then $\partial_{z} \theta>N$, and an overturning instability can develop generating convection locally in the flow. Figure 3 shows the probability density functions (PDFs) of the Eulerian $\mathrm{Ri}_{g}$ for all runs in isotropic domains. The PDFs of runs with $N=4\left(\mathrm{TG}_{1} 4\right.$ and $\left.\mathrm{RND}_{1} 4\right)$ display larger probabilities of low values of $\mathrm{Ri}_{g}(<1 / 4$ and $<0)$ than the runs with the same forcing but with $N=8$ ( $\mathrm{TG}_{1} 8$ and $\mathrm{RND}_{1} 8$ ). As $N$ is increased (for a given forcing), the peak of the PDF moves to larger values of $\mathrm{Ri}_{g}$. This indicates, as expected, that as stratification increases vertical instabilities are inhibited, and as a result we can also expect a less efficient vertical mixing (in agreement with the single-particle vertical dispersion observed in the previous section). However, when we compare TG and RND runs with the same value of $N$, we see that TG still shows larger probabilities of $\mathrm{Ri}_{g}<1 / 4$ and of $\mathrm{Ri}_{g}<0$. Indeed, the PDFs of the TG runs are shifted towards the left relative to the RND set, indicating that this flow is more vertically unstable and, consequently, can be more efficient at vertically mixing particles. 

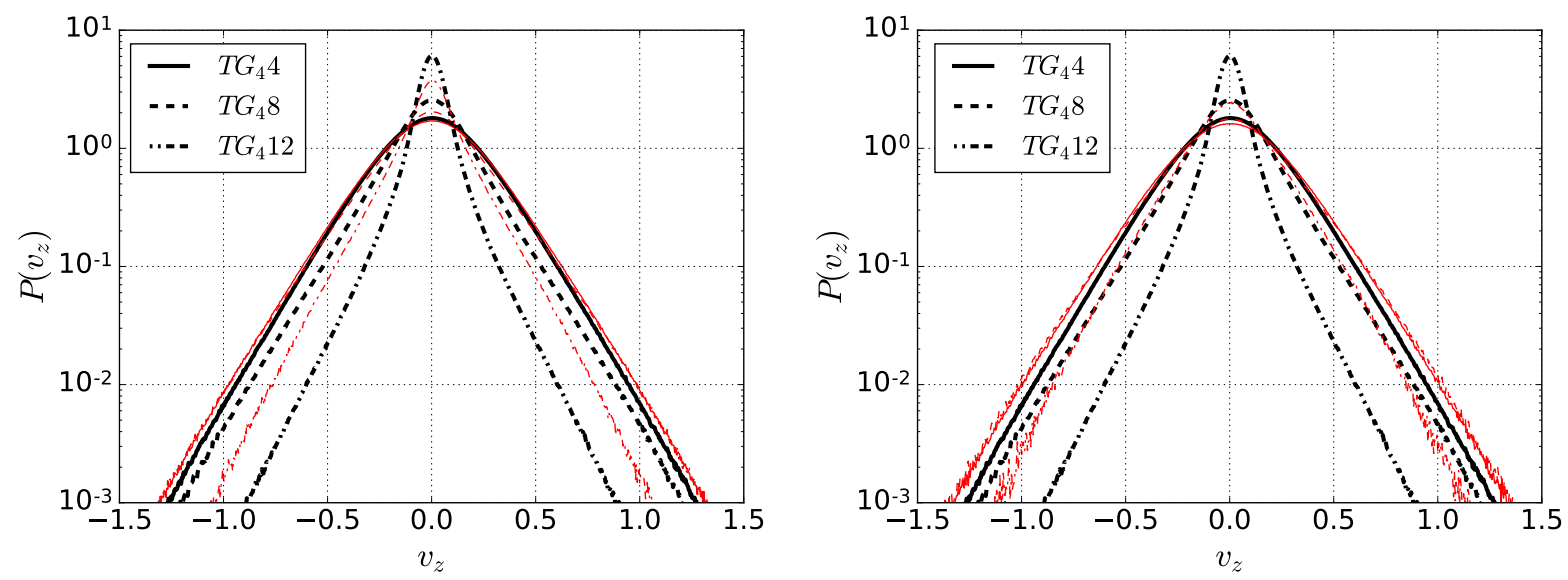

FIG. 6: (Color online). Both panels show in thick black lines the PDFs of the Lagrangian vertical velocity $v_{z}$ for TG runs in domains with 1:4 aspect ratio and varying $N$. PDFs restricted to particles in instants with $\mathrm{Ri}_{g}<1 / 4$ (left), or with $\mathrm{Ri}_{g}<0$

(right), for the same runs, are shown in (red) thin lines.

\section{B. The spatial structure of the local gradient Richardson number in the TG flow}

We are interested in how the TG forging affects the structure of the local gradient Richardson number. As the forcing generates a coherent large-scale flow, which in principle can affect vertical mixing, we show first in Fig. 4 the mean vertical value of the absolute Eulerian vertical velocity $\left\langle\left|u_{z}\right|\right\rangle_{z}$ computed for run $\mathrm{TG}_{4} 4$ (where the subscript $z$ in the brackets indicates the average was computed along the $z$-coordinate). As explained in Sec. III the TG flow consists of pairs of counter-rotating horizontal vortices, separated vertically by shear layers. Pressure gradients create a vertical circulation [38, and as a result the forcing generates a coherent structure at the largest scales that organize the flow into regions of high and low $\left\langle\left|u_{z}\right|\right\rangle$. As a result, some well-defined spatial regions in the flow display a bias towards larger values of $\left|u_{z}\right|$ (also associated with the generation of front- and filament-like structures in the flow, as discussed in [38]). It can be expected that Lagrangian particles approaching these regions will have a tendency to suffer larger displacements in the vertical, thus increasing $\delta z^{2}$ even at moderate $\mathrm{Rb}$.

To confirm this effect, Fig. 5 shows the PDFs of the Lagrangian $\mathrm{Ri}_{g}$ (i.e., now computed using the gradients as seen by the Lagrangian particles) for runs with TG forcing in the box with 1:4 aspect ratio. As observed before for the Eulerian statistics, as the stratification increases (i.e., for higher $N$ ) the mean gradient Richardson number also increases, and the fraction of fluid elements with $\mathrm{Ri}_{g}<1 / 4$ or $\mathrm{Ri}_{g}<0$ (i.e., prone to overturning) decreases. But the computation of $\mathrm{Ri}_{g}$ using the gradients as seen by the Lagrangian particles also allow us to compute conditional statistics, e.g., only for instants when the particles suffer large vertical velocities, or when the particles are in a specific region in space. Using the mean of the absolute Lagrangian vertical velocity $\left\langle\left|v_{z}\right|\right\rangle$ (averaged over all particles and over time), and the standard deviation of $v_{z}\left(\sigma_{v_{z}}\right)$, we computed the PDF of $\mathrm{Ri}_{g}$ restricted to particles with absolute vertical velocity $2 \sigma_{v_{z}}$ larger than $\left\langle\left|v_{z}\right|\right\rangle$ (see Fig. 5 ). With this restriction, the fraction of fluid elements that can suffer overturning instabilities increases (note the PDFs have a larger peak at $\mathrm{Ri}_{g}=0$, display larger values for $\mathrm{Ri}_{g}<0$, and smaller values for $\mathrm{Ri}_{g}>0$ when compared with the PDFs at the same $N$ without any restriction). This indicates that there is a correlation between fluid elements with $\mathrm{Ri}_{g} \leq 0$ and large values of $\left|v_{z}\right|$ (and thus, of particles displacing larger distances in the vertical, and thus contributing to $\delta z^{2}$ ). We also see that as $N$ is increased, the probability of finding fluid elements with $\mathrm{Ri}_{g} \leq 0$ decreases even when restricted to parcels with large $\left|v_{z}\right|$. Finally, Fig. 5 also shows the PDF of $\mathrm{Ri}_{g}$ restricted to the instants the particles are in the spatial regions of the large-scale circulation for which the largest absolute values of $u_{z}$ were observed in Fig. 4. A similar (albeit weaker) behavior as for the restriction in $v_{z}$ is found, with the shift in the peak of the PDFs towards smaller values of $\mathrm{Ri}_{g}$, confirming the relevance of the geometry of the large-scale flow in the TG runs in the vertical dispersion of Lagrangian particles.

To further study the effect of $\mathrm{Ri}_{g}$ on the vertical velocity of the particles, Fig. 6 shows the PDFs of the Lagrangian vertical velocity for all particles in TG runs with aspect ratio 1:4 and with varying $N$. As previously reported in [15, 16, the vertical velocity does not follow Gaussian statistics, and display strong tails (this feature is not exclusively associated with the TG forcing, as the same behavior was found in simulations with random forcing, see [15]). In [16] the extreme values were shown to be associated to intermittent overturning instabilities in the flow. Note that the behavior reported in [16] is non-monotonous in Fr, although for sufficiently small Fr (or sufficiently large values of $N$ ) the maximum values of $v_{z}$ decrease with increasing stratification (see Fig. 6). When we compute the PDFs restricted 

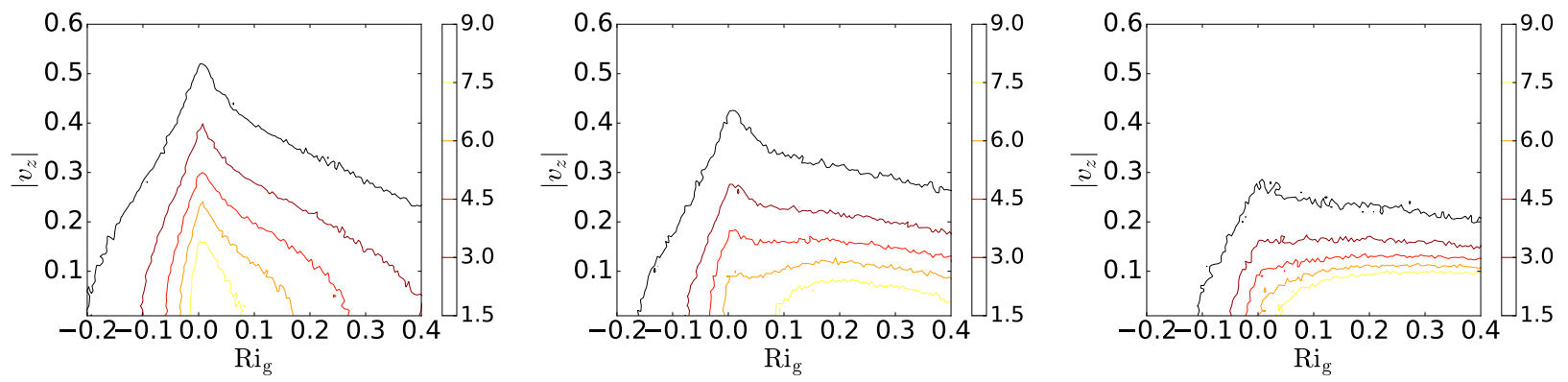

FIG. 7: (Color online). Isocontours of the joint probability distribution function of $\operatorname{Ri}_{g}$ and $\left|v_{z}\right|, P\left(\mathrm{Ri}_{g},\left|v_{z}\right|\right)$, for runs TG 4 (left), $\mathrm{TG}_{4} 8$ (right), and $\mathrm{TG}_{4} 12$ (left).
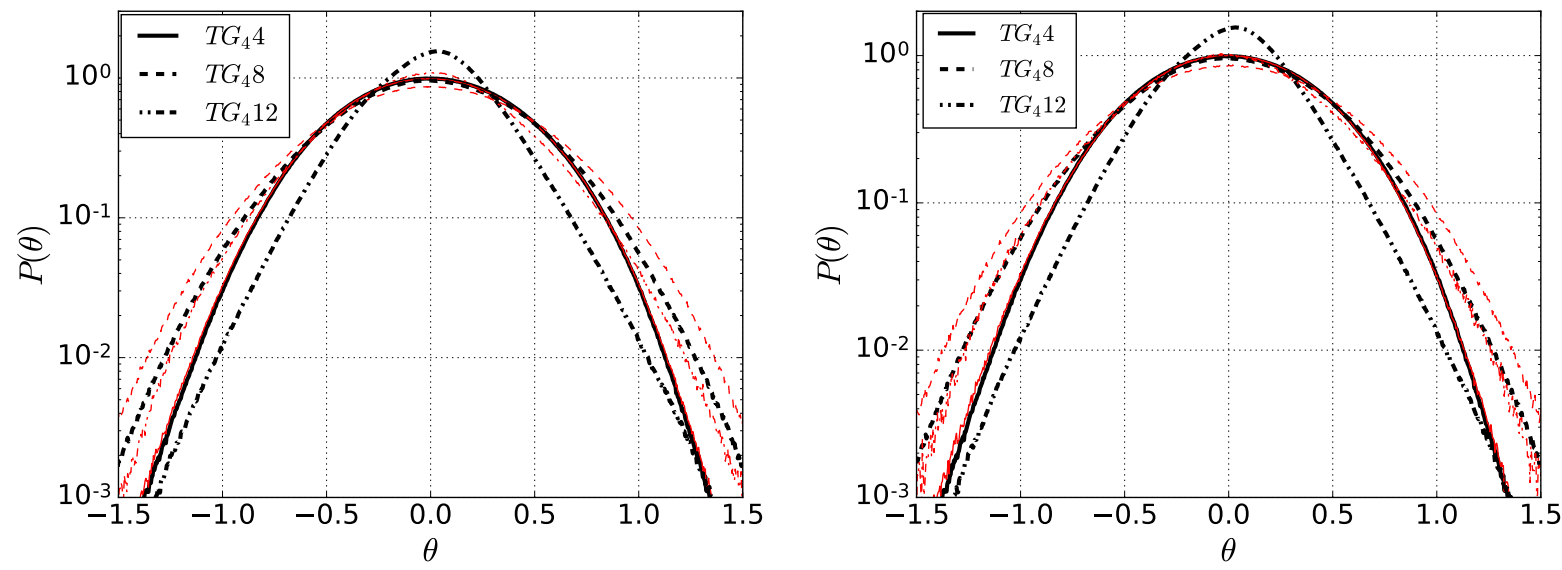

FIG. 8: (Color online). PDFs of $\theta$ as seen by the Lagrangian particles (thick black curves), and the same PDFs restricted (in thin red curves) to (left) particles at times with $\mathrm{Ri}_{g}<1 / 4$, and (right) particles at times with $\mathrm{Ri}_{g}<0$. Except for the run $\mathrm{TG}_{4} 12$, all PDFs are compatible with Gaussian statistics for $\theta$.

to particles in instants for which $\mathrm{Ri}_{g}<1 / 4$ or $\mathrm{Ri}_{g}<0$, while for the runs with moderate stratification $(N=4$ and 8$)$ there are only small changes in the tails of the PDFs (albeit extreme values of $v_{z}$ become more probable), for stronger stratification $(N=12)$ the changes are significantly larger, with stronger tails. This further confirms that points with $\mathrm{Ri}_{g}<1 / 4$ or $\mathrm{Ri}_{g}<0$ are associated with larger values of $v_{z}$, and can thus be expected to be associated with the enhanced dispersion after $t>2 \pi / N$ at least in the TG runs.

This can be confirmed in Fig. 7, which shows the joint probability density function as a function of $\operatorname{Ri}_{g}$ and $\left|v_{z}\right|$, $P\left(\mathrm{Ri}_{g},\left|v_{z}\right|\right)$, for the TG runs with aspect ratio 1:4 and with varying $N$. As the stratification increases, the probability of finding particles with large values of $\left|v_{z}\right|$ decreases, while that of finding larger values of $\mathrm{Ri}_{g}$ increases. For $N=4$ and $N=8$ note the correlation between larger absolute values of the vertical velocity with $\mathrm{Ri}_{g} \approx 0$ values, which is significantly weaker in the run with $N=12$.

Finally, we also studied how the value of $\operatorname{Ri}_{g}$ affects $\theta$ and $\partial_{z} \theta$ with increasing stratification (note that the local value of $\partial_{z} \theta$ is important for overturning instabilities, as the gradient of the buoyancy fluctuations can compete with the background gradient, resulting in local inversion of the stratification). Figure 8 shows the PDFs of $\theta$ as seen by the Lagrangian particles, and the same PDFs restricted to instants when $\mathrm{Ri}_{g}<1 / 4$ or $\mathrm{Ri}_{g}<0$, in all cases for the $T G_{4}$ runs. For $N=4$ the PDFs of $\theta$ are close to Gaussian, and the restriction in the values of $\mathrm{Ri}_{g}$ has a negligible effect in the statistics. However, for $N=8$, while the PDFs are still close to Gaussian, the restricted PDFs show a lower probability for $|\theta|<0.5$ and higher probability for $|\theta|>0.5$, indicating particles with $\mathrm{Ri}_{g}<0.25$ or $\mathrm{Ri}_{g}<0$ are more likely to be found in points with higher potential energy density $\sim \theta^{2}$. This behavior is enhanced for $N=12$, for which the PDFs also display non-Gaussian tails. Finally, Fig. 9 shows the PDFs of the Lagrangian vertical gradients of $\theta, \partial_{z} \theta$, which are non-Gaussian and asymmetric. The asymmetry is enhanced when the PDFs are restricted to instants when $\mathrm{Ri}_{\mathrm{g}}<1 / 4$ or $\mathrm{Ri}_{\mathrm{g}}<0$. While the non-restricted PDFs have their maximum at $\partial_{z} \theta \gtrsim 0$, for the restricted PDFs the maximum is at $\partial_{z} \theta \approx N$. From the ideal Boussinesq equation for $\theta$ (Eq. 2, with $\kappa=0$ ), it can be seen that $\boldsymbol{\nabla} \theta=(0,0, N)$ is a fixed point of both the equations for $\theta$ and for the Lagrangian evolution of $\partial_{z} \theta$, which could explain the accumulation of (restricted) particles with $\partial_{z} \theta \approx N$. Also, at points where $\operatorname{Ri}_{\mathrm{g}}<0$, then $\partial_{z} \theta>N$ (for 

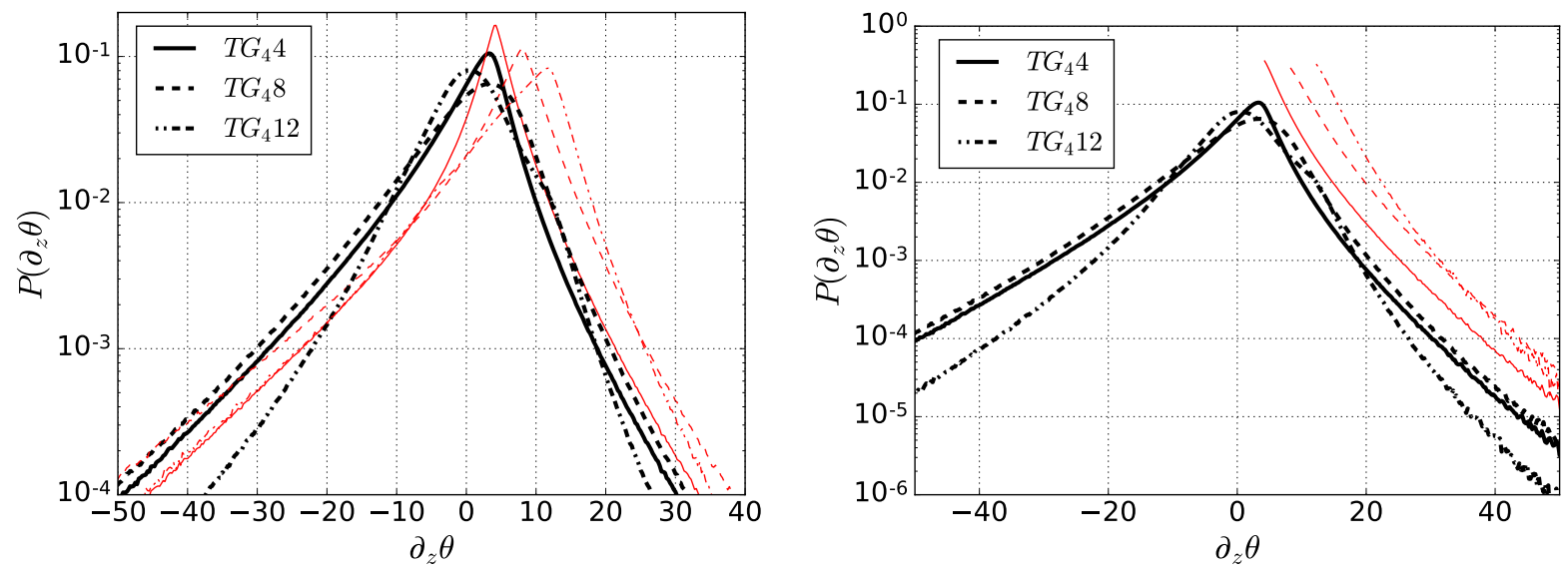

FIG. 9: (Color online). PDFs of the Lagrangian vertical temperature gradients $\partial_{z} \theta$ (thick black curves), and the same PDFs restricted (in thin red curves) to (left) $\mathrm{Ri}_{g}<1 / 4$ and (right) $\mathrm{Ri}_{g}<0$.

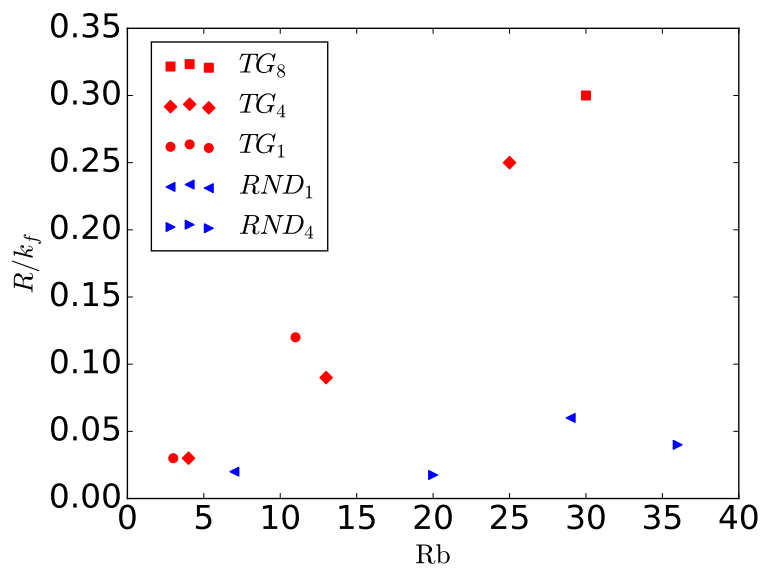

FIG. 10: (Color online). Overturning probability normalized by the forcing wave number, $R / k_{f}$, as a function of the Reynolds Buoyancy number $\mathrm{Rb}$ for all simulation in table $\mathrm{I}$.

which overturning events can occur). This is the reason why the PDFs of particles restricted to $\mathrm{Ri}_{\mathrm{g}}<0$ in Fig. 9 only take values of $\partial_{z} \theta$ greater than $N$.

\section{Overturning probability and the buoyancy Reynolds number}

As already mentioned, the extreme vertical velocities reported in the previous subsection are not exclusive of the TG flow. In [15, 16], non-Gaussian PDFs of $u_{z}, v_{z}$, and $\theta$ were reported for RND forcing depending on the values of $\mathrm{Fr}$ and $\mathrm{Rb}$. However, it is clear from the results shown so far that the geometry of the TG flow facilitates the development of overturning instabilities and the occurrence of extreme values of the vertical velocity even at moderate $\mathrm{Rb}$.

In the next section we will use these results to build a simple model for single-particle vertical dispersion, for all cases considered and independently of the two specific forcing function used. The results in Sec. III suggest that while the ballistic behavior of $\delta z^{2}$ for $t<2 \pi / N$ is dominated by the waves, the differences in $\delta z^{2}$ for $t>2 \pi / N$ depend on the strength of the vertical velocity and of the turbulence. For moderate turbulence (i.e., moderate values of $\mathrm{Rb}$ ) and without a large-scale vertical circulation, $\delta z^{2}$ is dominated by the waves even at late times, resulting in the observed saturation of the single-particle vertical dispersion. But for larger values of Rb (as in some of the RND runs), or in the presence of a large-scale flow (as in all TG runs), strong vertical updrafts or downdrafts can enhance vertical mixing resulting in the growth of $\delta z^{2}$ at late times. We will measure the probability of this happening by introducing an overturning probability $R$, defined as the fraction of particles (in the Lagrangian frame) or the fraction of space 

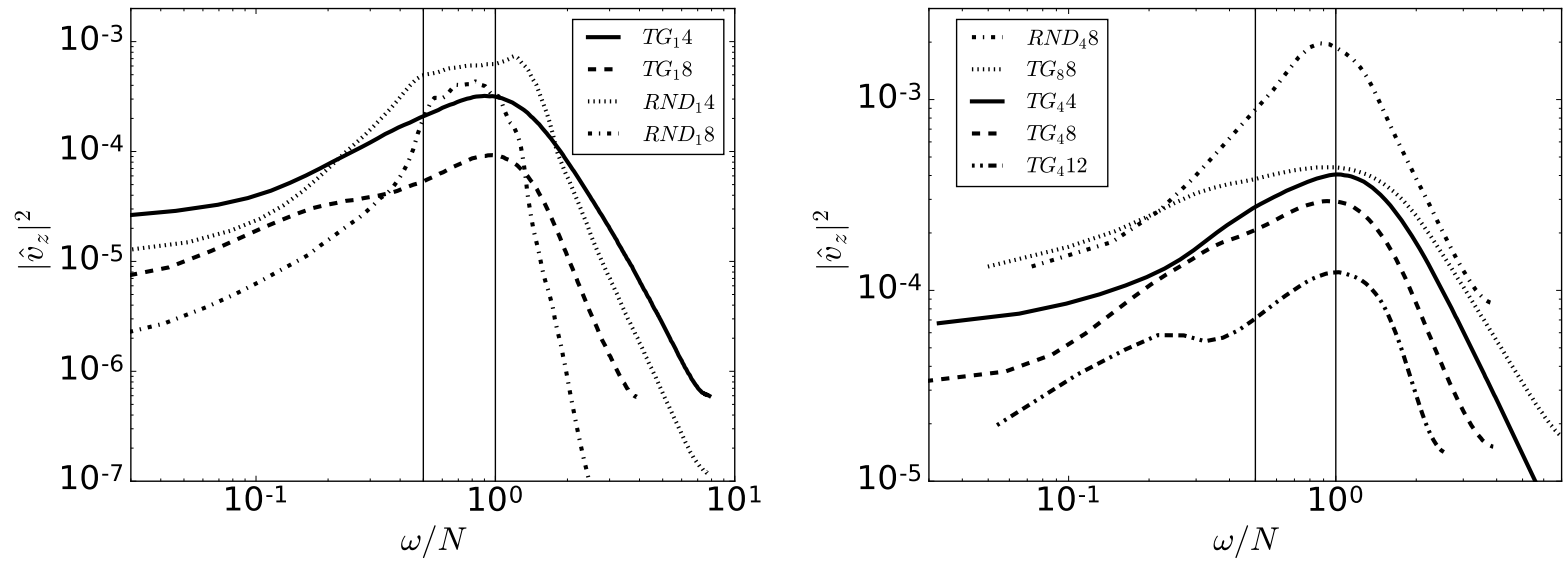

FIG. 11: Power spectrum of the Lagrangian vertical velocity for (left) runs in isotropic domains, and (right) runs in elongated domains. Frequencies have been normalized by the Brunt-Väisälä frequency. The solid vertical lines indicate (from left to right) $\omega=N / 2$ and $\omega=N$.

volume (in the Eulerian frame) with $\mathrm{Ri}_{g}<0$. Figure 10 (see also table $\mathbb{I}$ ) gives $R / k_{f}$ as a function of $\mathrm{Rb}$ for all runs, where $R$ is measured as the integral of the $\mathrm{PDF}$ of $\mathrm{Ri}_{g}$ for $\mathrm{Ri}_{g}<0$. The data follows (for the range of $\mathrm{Rb}$ considered) a linear relation with $\mathrm{Rb}$, with two different slopes for the TG and RND runs (even when the runs in each set also have different aspect ratios, forcing wavenumbers, Reynolds, and Froude numbers). As expected, for fixed Rb, the TG runs display larger values of $R$ than the RND runs.

\section{SINGLE-PARTICLE VERTICAL DISPERSION MODEL}

To study the vertical dispersion of single-particles observed in the DNSs of SST in section III, we now present a stochastic model that combines a random wave model (to consider the effect of internal gravity waves) with a CTRW [35] (to capture the effect of overturning by turbulent or large-scale eddies).

The wave model consists in a sum of linear waves with random phases. A Lagrangian particle moving vertically following these waves has a trajectory

$$
z_{\text {wav }}(t)=\Re\left[\sum_{\omega} A_{\omega} e^{i\left(\omega t+\phi_{\omega}\right)}\right],
$$

where $\phi_{\omega}$ is a random phase, and where the sum is performed over $N_{\omega}$ uniformly distributed frequencies in the range of frequencies $\omega \in\left[\omega_{\min }, \omega_{\max }\right]$. The amplitude of the waves satisfies the spectral relation

$$
A_{\omega}=A_{0} \omega^{-1}
$$

for the same range of frequencies, and where $A_{0}$ is a normalization factor. The dependence of $A_{\omega} \sim \omega^{-1}$ follows from observations that the power spectrum of the actual Lagrangian vertical velocity has a broad maximum with approximately constant amplitude near the Brunt-Väisälä frequency. Note that associating $v_{z}$ with $\dot{z}_{\text {wav }}=\Re\left[\sum A_{\omega} \omega e^{i\left(\omega t+\phi_{\omega}\right)}\right]$, a flat power spectrum for $v_{z}$ implies Eq. (13) for the amplitude of the waves. Once $N_{\omega}$ is chosen, the normalization factor $A_{0}$ can then be fixed as $A_{0}=\left(2 U_{z}^{2} / N_{\omega}\right)^{1 / 2}$ by imposing that for each particle $\left\langle\dot{z}_{\text {wav }}^{2}\right\rangle_{t}$ (averaged over time) must be equal to the mean squared Eulerian vertical velocity $U_{z}^{2}$ (also equal to the mean squared Lagrangian vertical velocity), using Parseval's theorem.

Note that a flat Lagrangian spectrum for a range of frequencies is compatible with oceanic observations of the so-called Garrett-Munk spectrum, and also with numerical simulations of SST [11. As an example, Fig. 11, shows the power spectrum of the Lagrangian vertical velocity for all runs in table 1 . There is a broad peak near $\omega=N$, and in several of the runs an approximately flat spectrum can be observed in its vicinity (as a reference, the figure indicates a range of frequencies $[N / 2, N]$ ), with a decay compatible with a power law for $\omega>N$, and a slowly decaying, or almost flat, spectrum for $\omega \ll N)$. Also, for the runs with the smallest values of Rb considered in this study (runs $\mathrm{TG}_{1} 8$ with $\mathrm{Rb}=3.2$, and $\mathrm{TG}_{4} 12$ with $\mathrm{Rb}=4.3$ ), a secondary peak at smaller frequencies can be observed. In these runs turbulence is moderate, and the waves dominate the dispersion at intermediate times. 


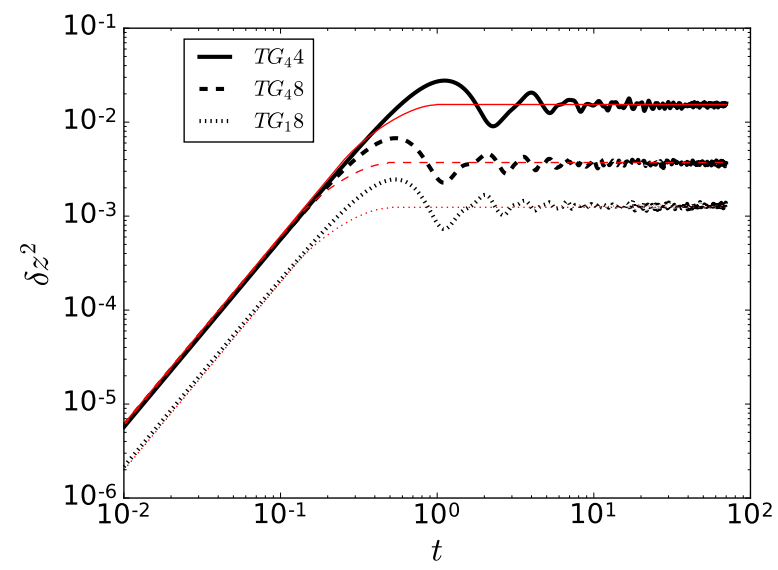

FIG. 12: (Color online). Mean dispersion obtained from a random superposition of waves as in Eq. 14 (in thick black lines), and from Eq. (15) (in thin red lines), with the parameter $A_{0}$ to match the Eulerian vertical r.m.s. velocity and $N$ the Brunt-Väisälä frequency for some of the TG runs (see labels in the inset).

As the dispersion relation of internal gravity waves is $\omega=N k_{\perp} / k \leq N$, we set $\omega_{\max }=N$, and for simplicity, from the results in Fig. 11 we set $\omega_{\min }=N / 2$ in all cases. It then follows from Eq. (12) that the vertical displacement of any particle following the waves is given by

$$
\delta z_{w a v}(t)=z_{w a v}(t)-z_{w a v}(0)=\sum_{\omega} A_{w}\left[\cos \left(\omega t+\phi_{\omega}\right)-\cos \left(\phi_{\omega}\right)\right] .
$$

The square of this expression, when averaged over an ensemble of particles and waves, can be approximated by (see Appendix A

$$
\left\langle\delta z_{\text {wav }}^{2}\right\rangle(t)=\left\{\begin{array}{ccc}
U_{z}^{2} t^{2} & \text { if } & t \leq N^{-1}, \\
P(t) & \text { if } & N^{-1}<t<4 N^{-1}, \\
\frac{4 U_{z}^{2}}{N^{2}} & \text { if } & t \geq 4 N^{-1},
\end{array}\right.
$$

where $P(t)$ is a third order polynomial function obtained by imposing $\left\langle\delta z_{\text {wav }}^{2}\right\rangle$ and its time derivative to be continuous in time (see Appendix A). Figure 12 shows the mean dispersion for many particles calculated from a stochastic superposition of waves as in Eq. (14), and from the function in Eq. (15), in both cases using values of $A_{0}$ and $N$ that adjust the vertical r.m.s. velocity and the Brunt-Väisälä frequency of several TG runs. The function in Eq. (15) is in good agreement with the sum of random waves, specially for short and long times. Note also that this simple model based on a superposition of waves captures the early-time ballistic behavior of $\delta z^{2} \sim t^{2}$ seen for all DNSs in Fig. 1 , as well as the saturation at late times seen in Fig. 1 for some of the simulations.

The behavior shown in Fig. 12 is similar to the vertical dispersion predicted for SST by other models based on a linear superposition of waves [28], and is reminiscent of the vertical dispersion observed in previous DNSs of SST at moderate values of $\mathrm{Rb}[11,29$. However, this wave model fails to reproduce the dispersion observed at long times in some of our runs. To introduce an enhanced vertical dispersion by turbulent overturning, we add a CTRW model that mimics the trapping of particles by eddies, resulting in vertical displacements when the flow becomes unstable such that the total vertical dispersion will be $\delta z(t)=\delta z_{\text {wav }}(t)+\delta z_{C T R W}(t)$.

To compute $\delta z_{C T R W}(t)$, in each step $t$ of the random walk process we assume a particle has a probability $R$ of being trapped for a time $t_{t}$ inside an eddy of radius $r_{t}$ with velocity $U_{t}$. As in the previous section, $R$ is the probability of finding particles with $\mathrm{Ri}_{g}<0$. The probability of finding an eddy of radius $r_{t}$ is given by a Kolmogorov distribution $P\left(r_{t}\right) \sim r_{t}^{4 / 3}$ for $r<L_{o z}$, compatible with an isotropic energy spectrum $\sim k^{-5 / 3}$ for wavenumbers $k>k_{o z}$; in other words, we assume that the eddies responsible for the vertical dispersion at late times are associated with overturning instabilities in the turbulent inertial range for scales equal to or smaller than the Ozmidov scale. The distribution of trapping times $P\left(t_{t}\right)$ is continuous and uniform between $t_{t}=0$ and the Eulerian turnover time at the Ozmidov scale $\tau_{o z}$. Finally, the distribution of velocities of the eddies $P\left(U_{t}\right)$ is the observed PDF of the absolute value of the 

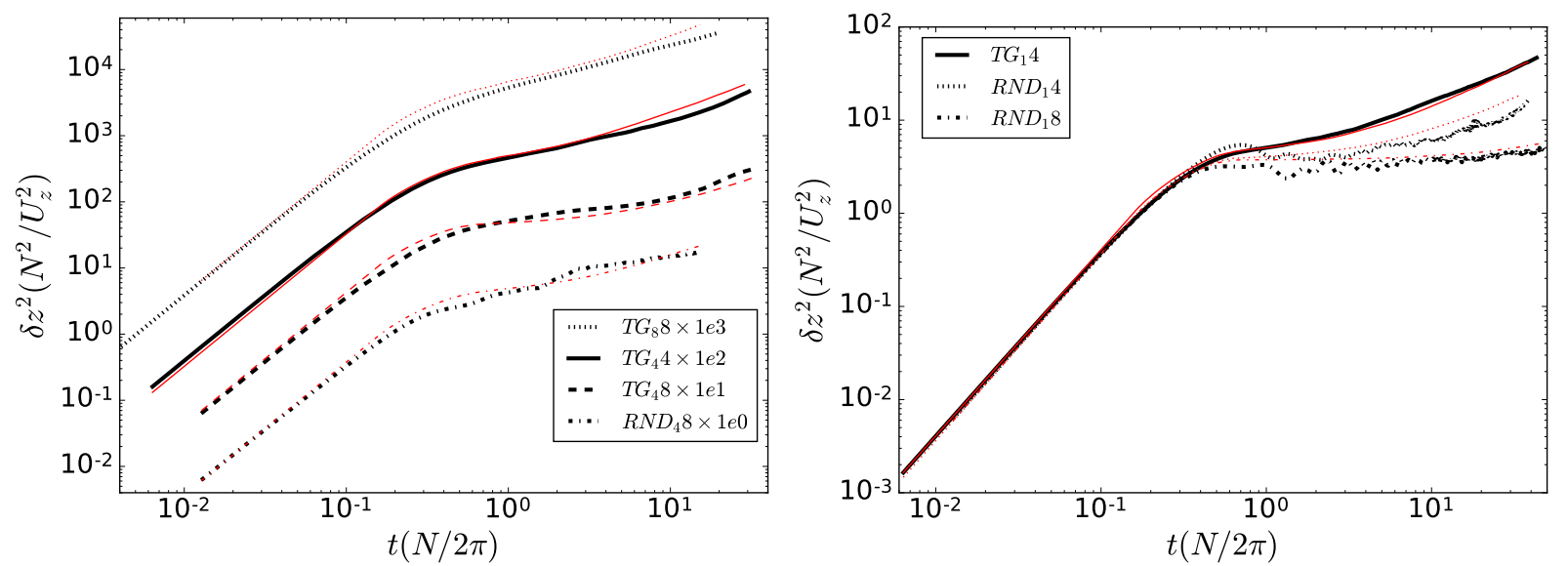

FIG. 13: (Color online). Mean vertical dispersion $\delta z^{2}$ for (left) runs in elongated domains with amplitudes rescaled for better visualization (see the inset), and (right) runs in isotropic domains, for RND and TG forcing, and with different values of $N$. Normalizations of $\delta z^{2}$ and of time are the same as in Fig. 1 In both figures the thick (black) lines show the results from the DNSs, and the thin (red) lines show the results obtained from the single-particle dispersion model.

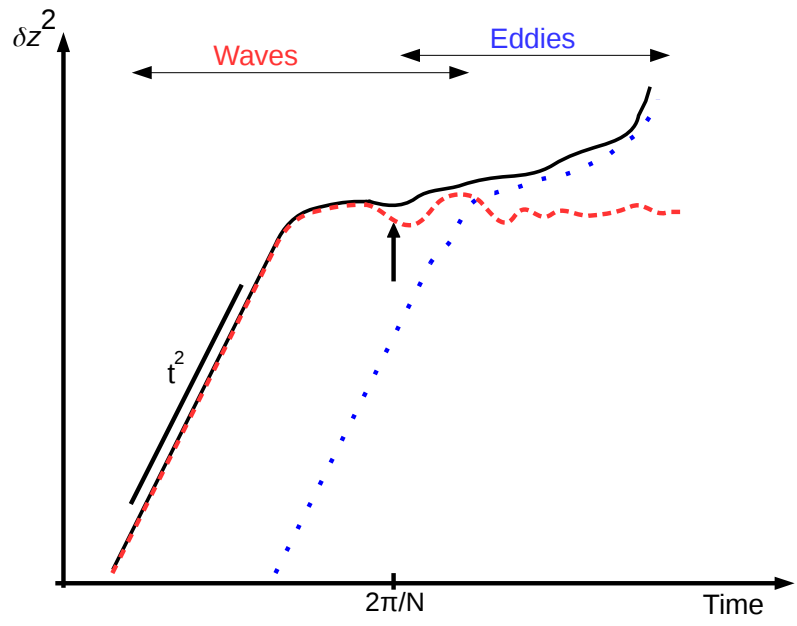

FIG. 14: (Color online). Sketch of the two contributions to vertical dispersion in the model. The random superposition of waves dominates at early times (red dashed curve), while the effect of turbulent eddies and overturning events become relevant at late times (blue dotted curve). The total vertical dispersion (black solid curve) results from the superposition of both effects.

Eulerian vertical velocity (which, in practice, can be very well approximated by assuming that it follows a Rayleigh distribution, so knowledge of the r.m.s. value of $u_{z}, U_{z}$, is sufficient to estimate $P\left(U_{t}\right)$ ).

In each step of the CTRW, if a particle is not trapped by an eddy, $\delta z_{C T R W}(t)$ will remain constant (i.e., the particle will not move as a result of eddy trapping). If it gets trapped, it will be displaced along a circle as the result of the trapping, with a vertical displacement of $r_{t} \sin \left(\theta_{t}\right)$ which is just the projection of the circular trajectory of radius $r_{t}$ in the $z$ direction, and where $\theta_{t}=U_{t} t_{r} / r_{t}$ is the angle of the arc traveled during the time $t_{r}$. Thus, the random walk process just mimics in a simplified way the eventual presence of vertical eddies that can result in upward or downward transport of the Lagrangian particles. As described above the model has no free parameters; all parameters are obtained from Eulerian characteristic lengths and time scales of the flow.

Figure 13 shows the mean squared vertical dispersion obtained from several DNSs, and $\delta z^{2}(t)=\left[\delta z_{\text {wav }}(t)+\right.$ $\left.\delta z_{C T R W}(t)\right]^{2}$ as obtained from the wave and CTRW model. For the runs in elongated domains (with TG forcing, or larger values of $\mathrm{Rb}$ ), as the dispersion is very similar for all runs, we rescaled $\delta z^{2}$ using an arbitrary value (indicated in the figure inset), so the curves can be distinguished more easily. In other cases, the same normalization as in Fig. 1 was used for $\delta z^{2}$ and time. The model is in good agreement with the DNS data in all cases, and captures early and late time behavior independently of the forcing function (TG or RND), as well as cases with saturation of $\delta z^{2}(t)$ 

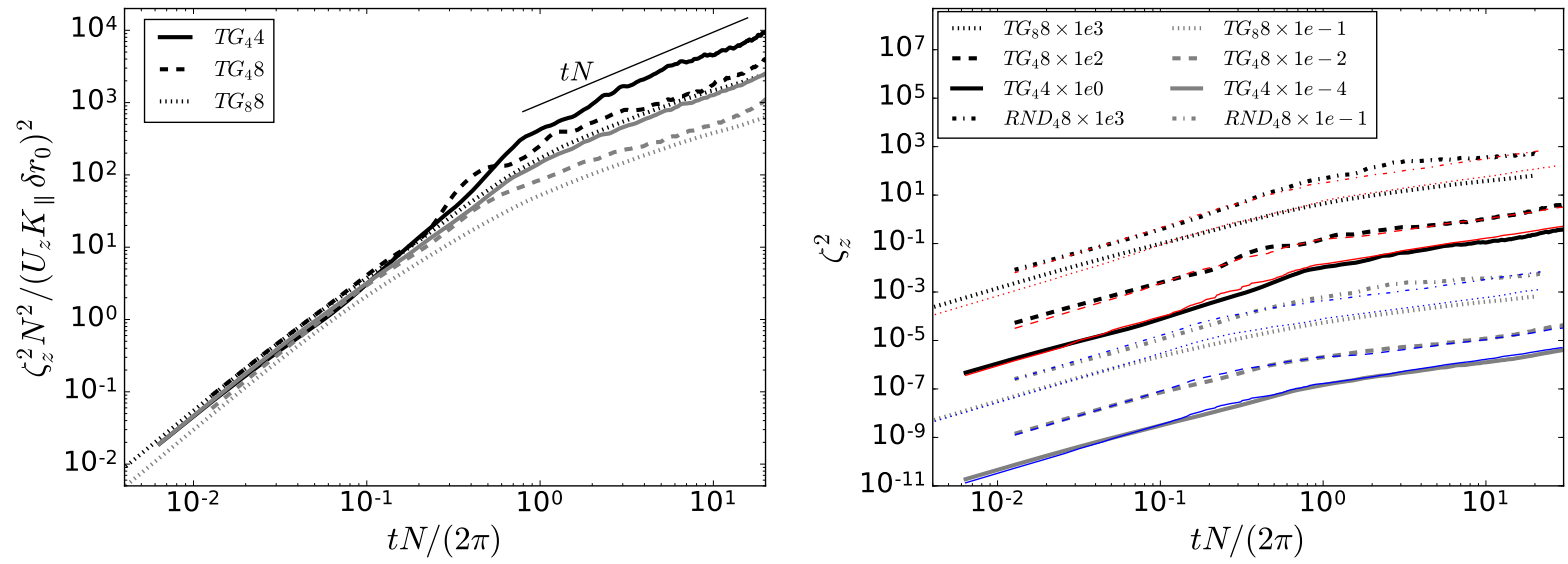

FIG. 15: Color online). Left: two-particle vertical dispersion for particles with initial vertical separation $\delta z_{0} \ll \eta$, and two initial horizontal separations: $\delta r_{0}=\eta$ (black lines) $\delta r_{0}=2 \eta$ (gray lines). Results from three simulations with TG forcing are shown. The vertical dispersion was normalized by $\left(w K_{\|} \delta r_{0}\right)^{2} / N^{2}$, and time by $1 / N$. With this normalization all curves

collapse during the ballistic regime. A power law at later times is indicated as a reference. Right: two-particle vertical dispersion from DNSs with RND or TG forcing (thick lines), and from the model (thin lines). The amplitude of the curves have been rescaled for better visualization (see the rescaling factor in the inset).

for $t>2 \pi / N$ as cases in which $\delta z^{2}(t)$ keeps growing at late times. The differences in these two behaviors can now be further clarified by the model (see Fig. 14). At early times, the waves dominate the dispersion resulting in the observed ballistic regime up to the period of the slowest waves, $t \lesssim 2 \pi / N$, for which the largest "fast" displacements (on the time scale of the waves) can take place. If considered alone, trapping by turbulent eddies in the CTRW model would also result in ballistic growth of $\delta z^{2}$, but it has an initial value significantly smaller, and as a result this process is subdominant to the dispersion by the waves. At intermediate times $(t \approx 2 \pi / N)$ dispersion by the waves saturates generating the plateau. If turbulence is moderate (and thus $R$ is also moderate), trapping by eddies is inefficient, resulting in a temporary saturation of the dispersion, or, in the most extreme cases, in a complete saturation of $\delta z^{2}$. Depending on how strong the turbulence is, at a certain time overturning events can start enhancing the dispersion, and for longer times the turbulence dominates the dynamic surpassing the effect of the waves.

\section{TWO-PARTICLE VERTICAL DISPERSION}

We can also study the two-particle vertical dispersion $\zeta_{z}^{2}$, which the two simple processes presented above (dispersion by a random superposition of waves, and a CTRW process to capture the effect of turbulent overturning events) can also model. The two-particle vertical dispersion is defined as $\zeta_{z}^{2}=\left\langle\left[z_{i}(t)-z_{j}(t)\right]^{2}\right\rangle_{(i, j)}$, where $i \neq j$ are the labels of two particles that at the initial time have a vertical separation $\delta z_{0}$ and a horizontal separation $\delta r_{0}$, and where the subindex $(i, j)$ denotes that the average is computed over pairs of particles. Figure 15 shows the resulting two-particle vertical dispersion for runs $\mathrm{TG}_{4} 4, \mathrm{TG}_{4} 8$, and $\mathrm{TG}_{8} 8$. We consider pairs of particles which at time $t=0$ have a vertical separation $\delta z_{0} \ll \eta$ (where $\eta$ is the Kolmogorov dissipation scale), and horizontal separations $\delta r_{0} \approx \eta$ or $\delta r_{0} \approx 2 \eta$ (see 29] for a detailed study on different choices of the initial separation in two-particle dispersion in SST). Normalizing the vertical dispersion $\zeta_{z}^{2}$ by $\left(U_{z} K_{\|} \delta r_{0}\right)^{2} / N^{2}$ all curves approximately collapse during the ballistic regime. As for the case of single-particle dispersion, we see again a growth of the two-particle dispersion at late times, which is linear or almost linear with $t$ in all runs. Here we also see an effect of Rb: simulations with larger Rb display larger two-particle vertical dispersions at late times. It is also worth pointing out that when the initial horizontal separation $\delta r_{0}$ is increased (for a given run), the short-time two-particle dispersion augments proportionally, but the late-time two-particle dispersion remains equal, indicating a decorrelation of the two particles at late times as reported before in [29] (note that in Fig. 15, as $\zeta_{z}^{2}$ is normalized by $\delta r_{0}^{2}$, this late-time decorrelation results in different amplitudes of $\left(\zeta_{z} / \delta r_{0}\right)^{2}$ as $\delta r_{0}^{2}$ is changed).

As mentioned above, the two-particle vertical dispersion can be modeled using an extension of the single-particle model. As before, we consider the effect of the waves and of the turbulent eddies separately. First, if we have two particles which are initially very close to each other (almost at the same height, but with a horizontal displacement $\delta r_{0}$ ), we can assume they will be displaced by the same waves but with a phase difference between the two (for each 
wave with frequency $\omega)$ given by

$$
\phi_{\omega}^{\prime} \approx \phi_{\omega}+k \delta r_{0}
$$

Here $\phi_{\omega}$ is the phase of the wave seen by one of the particles, $\phi_{\omega}^{\prime}$ is the phase seen by the other particle, $k$ is the wavenumber, and we approximate the total separation between the two particles by $\delta r_{0}$ (as $\delta z_{0} \ll \eta$ ). Using the expressions for the displacements of a particle in a superposition of random waves given by Eqs. (12) and (14), we can estimate the separation as a function of time for a single pair as

$$
\zeta_{i j,(w a v)}(t)=\sum_{\omega} A_{\omega}\left\{\left[\cos \left(\omega t+\phi_{\omega}\right)-\cos \left(\phi_{\omega}\right)\right]-\left[\cos \left(\omega t+\phi_{\omega}^{\prime}\right)-\cos \left(\phi_{\omega}^{\prime}\right)\right]\right\}+\delta z_{0}
$$

where the subindexes $i$ and $j$ again label the pairs of particles that at the initial time meet the condition $\delta r_{0} \approx \eta$ $($ or $\approx 2 \eta)$. Equation $(17)$ is just the difference between two single-particle vertical trajectories, to which we added an initial vertical separation $\delta z_{0} \ll \eta$. As we did in the previous section, the resulting dispersion, when averaged over several pairs of particles and sets of random waves, can be approximated as (see Appendix B

$$
\left\langle\zeta_{z,(\text { wav })}^{2}\right\rangle(t) \approx \begin{cases}U_{z}^{2}\left(2.1 \delta r_{0} \frac{2 \pi}{L_{\|}}\right)^{2} t^{2} & \text { if } \quad t<N^{-1}, \\ Q(t) & \text { if } N^{-1}<t<4 N^{-1}, \\ \frac{16 U_{z}^{2}\left(2.1 \delta r_{0} \pi\right)^{2}}{\left(L_{\|} N\right)^{2}} & \text { if } \quad t \geq 4 N^{-1},\end{cases}
$$

where, as in the single-particle approximation in Eq. (15), $Q(t)$ is a third order polynomial interpolation obtained by imposing the function and its derivative to be continuous in time, and we neglected all terms in $\delta z_{0}$ and $\delta z_{0}{ }^{2}$ as they are very small when compared with the leading order terms.

As at late times the particles separate significantly from each other, to take into account the effect of overturning we can assume the two particles are uncorrelated, and as a result we can just consider two independent CTRW processes with the same properties as the one described in the previous section for single-particles, one for each particle in the pair. This is compatible with observations of two-particle dispersion in DNSs of SST [29], and with the results from DNSs shown above, indicating the late time dispersion becomes independent of the original separation $\delta r_{0}$. The final result of combining $\zeta_{z, \text { (wav) }}$ with the CTRW processes is shown in Fig. 15. The model is in good agreement with the two-particle vertical dispersion obtained from the DNSs, both in the ballistic regime as well as for long times when dispersion becomes dominated by the turbulent eddies, for both forcing functions considered, different domain aspect ratios, and different values of Fr and $\mathrm{Rb}$.

\section{CONCLUSIONS}

In this paper we studied single- and two-particle vertical dispersion for Lagrangian trajectories in forced stably stratified turbulence, using two different forcing functions (Taylor-Green and random forcing), domains with different aspect ratios, and different Froude and Reynolds numbers. Using direct numerical simulations we showed that latetime saturation of single-particle vertical dispersion, reported in previous studies of this problem, is obtained only for moderate values of the buoyancy Reynolds number, and that for larger values of Rb, or even for moderate Rb in the case of the Taylor-Green flow that develops a vertical circulation, the saturation does not take place. Instead, $\delta z^{2}$ keeps growing in time after the ballistic regime, albeit at a slower rate than in homogeneous and isotropic turbulence.

We showed that the gradient Richardson number plays an important role in the strength of the vertical mixing of Lagrangian tracers, as overturning fluid elements with $\mathrm{Ri}_{g}<0$ give an important contribution to vertical displacement of Lagrangian particles. In particular, regions of the flows with higher vertical velocity present a higher probability of having particles with $\mathrm{Ri}_{g}<0$ and vice versa. The joint probability (or restricted PDFs) between $\mathrm{Ri}_{g}$ and the Lagrangian vertical velocity, temperature fluctuations and gradients were studied, confirming this correlation.

Based on these results, we derived models for single- and two-particle dispersion that consist of a superposition of random waves (to capture the early time ballistic regime), and an eddy-constrained continuous-time random walk process (to capture the effect of turbulent eddies and overturning instabilities in the flow at late times). The dispersion obtained from these models is in good agreement with the vertical dispersions obtained from the direct numerical simulations. This agreement strengthens the observation that the waves dominate the dynamic of particles at short times, resulting in the initial ballistic regime, while at intermediate times $(t \approx 2 \pi / N)$ linear and non-linear effects 
coexist in the dynamics, giving rise to a transient that can develop (or not) a plateau on the dispersion depending on how strong (or weak) is the effect of overturning events. At later times, and if turbulence is sufficiently strong (as measured by $\mathrm{Rb}$, or equivalently, by the probability of a fluid element to suffer overturning, $R=P\left(\mathrm{Ri}_{g}<0\right)$ ), turbulence (modeled here by the continuous-time random walk process) dominates.

The superposition of linear and turbulent contributions to the dispersion in the model thus allows clarification of the relevant time and length scales involved in the dynamics of Lagrangian tracers in stratified turbulence. Finally, as all parameters in the model can be obtained from large-scale Eulerian properties of the flow, the model opens the door to modeling turbulent dispersion of tracers in Eulerian simulations of stratified flows that do not resolve the smallest scales in the flow, as usually is the case in the study of atmospheric and oceanic flows.

\section{Appendix A: Derivation of the single-particle dispersion wave model}

We want to derive averaged expressions for the dispersion as a function of time resulting from a random superposition of waves as that given by Eq. (14). For short times, from

$$
\delta z_{w a v}(t)=\sum_{\omega} A_{w}\left[\cos \left(\omega t+\phi_{\omega}\right)-\cos \left(\phi_{\omega}\right)\right]
$$

we can take the square, use the trigonometric identity $\cos \left(\omega t+\phi_{\omega}\right)=\cos (\omega t) \cos \left(\phi_{\omega}\right)-\sin (\omega t) \sin \left(\phi_{\omega}\right)$, the Taylor expansions to first order $\sin (\omega t) \approx \omega t$ and $\cos (\omega t) \approx 1$, and Eq. 13 with $A_{0}=\left(2 U_{z}^{2} / N_{\omega}\right)^{1 / 2}$, to get

$$
\delta z_{\text {wav }}^{2} \approx t^{2} \frac{2 U_{z}^{2}}{N_{\omega}}\left[\sum_{\omega} \sin \left(\phi_{\omega}\right)\right]\left[\sum_{\omega^{\prime}} \sin \left(\phi_{\omega^{\prime}}\right)\right]=t^{2} \frac{2 U_{z}^{2}}{N_{\omega}}\left[\sum_{\omega} \sin ^{2}\left(\phi_{\omega}\right)+\sum_{\omega, \omega^{\prime} \neq \omega} \sin \left(\phi_{\omega}\right) \sin \left(\phi_{\omega^{\prime}}\right)\right] .
$$

As the average over random phases $\phi$ uniformly distributed between 0 and $2 \pi$ is

$$
\begin{gathered}
\langle\sin (\phi)\rangle_{\phi}=\langle\cos (\phi)\rangle_{\phi}=0, \\
\left\langle\sin ^{2}(\phi)\right\rangle_{\phi}=\left\langle\cos ^{2}(\phi)\right\rangle_{\phi}=1 / 2,
\end{gathered}
$$

for $\phi_{\omega}$ and $\phi_{\omega^{\prime}}$ two independent stochastic variables, for short times and after averaging over an ensemble of particles with different sets of random waves, we then have

$$
\left\langle\delta z_{\text {wav }}^{2}\right\rangle(t) \approx t^{2} U_{z}^{2}
$$

For long times

$$
\delta z_{\text {wav }}^{2}(t)=\frac{2 U_{z}^{2}}{N_{\omega}}\left\{\sum_{\omega} \frac{1}{\omega}\left[\cos \left(\omega t+\phi_{\omega}\right)-\cos \left(\phi_{\omega}\right)\right]\right\}\left\{\sum_{\omega^{\prime}} \frac{1}{\omega^{\prime}}\left[\cos \left(\omega^{\prime} t+\phi_{\omega^{\prime}}\right)-\cos \left(\phi_{\omega^{\prime}}\right)\right]\right\},
$$

which can be rewritten as

$\delta z_{\text {wav }}^{2}(t)=\frac{2 U_{z}^{2}}{N_{\omega}}\left\{\sum_{\omega} \frac{1}{\omega^{2}}\left[\cos \left(\omega t+\phi_{\omega}\right)-\cos \left(\phi_{\omega}\right)\right]^{2}+\sum_{\omega, \omega^{\prime} \neq \omega} \frac{1}{\omega \omega^{\prime}}\left[\cos \left(\omega^{\prime} t+\phi_{\omega^{\prime}}\right)-\cos \left(\phi_{\omega^{\prime}}\right)\right)\left(\cos \left(\omega t+\phi_{\omega}\right)-\cos \left(\phi_{\omega}\right)\right]\right\}$.

As the time average over several wave periods results in $\left\langle\cos \left(\omega t+\phi_{\omega}\right)^{2}\right\rangle_{t}=1 / 2,\left\langle\cos \left(\omega t+\phi_{\omega}\right)\right\rangle_{t}=0$, and $\left\langle\cos \left(\omega t+\phi_{\omega}\right) \cos \left(\omega^{\prime} t+\phi_{\omega^{\prime}}\right)\right\rangle_{t}=0$, using Eqs. A3 and A4, we obtain the average of Eq. A7 over time and over an ensemble of particles and random waves as

$$
\left\langle\delta z_{w a v}^{2}\right\rangle_{t} \approx \frac{2 U_{z}^{2}}{N_{\omega}} \sum_{\omega=\omega_{\min }}^{N} \frac{1}{\omega^{2}}
$$

Using $\Delta \omega=\left(N-\omega_{\min }\right) / N_{\omega}$, then

$$
\sum_{\omega=\omega_{\min }}^{N} \frac{1}{\omega^{2}}=\sum_{\omega=\omega_{\min }}^{N} \frac{1}{\omega^{2}} \frac{\Delta \omega}{\Delta \omega} \approx \frac{1}{\Delta \omega} \int_{\omega_{\min }}^{N} \frac{1}{\omega^{2}} d \omega=\frac{1}{\Delta \omega} \frac{N-\omega_{\min }}{N \omega_{\min }}=\frac{N_{\omega}}{N \omega_{\min }},
$$


and finally, Eq. A8] can we rewritten as

$$
\left\langle\delta z_{\text {wav }}^{2}\right\rangle_{t} \approx \frac{2 U_{z}^{2}}{N \omega_{\min }}
$$

where we chose $\omega_{\min }=N / 2$.

This gives the early time $\left(t \leq N^{-1}\right)$ and late time $\left(t \geq 4 N^{-1}\right)$ expressions in Eq. 15 (note the choices of $N^{-1}$ and $4 N^{-1}$ as the two limits for the validity of the approximations are somewhat arbitrary). To obtain a smooth (i.e., continuous in $\left\langle\delta z_{\text {wav }}^{2}\right\rangle(t)$ and in its time derivative) interpolation between these two expressions, we use a third order polynomial function to interpolate $\left\langle\delta z_{\text {wav }}^{2}\right\rangle(t)$ between $t_{a}=1 / N$ and $t_{b}=4 / N$. Writing a polynomial approximation $\left\langle\delta z_{\text {wav }}^{2}\right\rangle(t)=P(t)=A t^{3}+B t^{2}+C t+D$, then the coefficients after imposing the continuity conditions are

$$
\begin{aligned}
& A=\left[P^{\prime}\left(t_{a}\right)\left(t_{a}-t_{b}\right)-2\left(P\left(t_{a}\right)-P\left(t_{b}\right)\right)\right] /\left(t_{a}-t_{b}\right)^{3}, \\
& B=-\left[P^{\prime}\left(t_{a}\right)\left(t_{a}^{2}+t_{a} t_{b}-2 t_{b}^{2}\right)+3\left(t_{a}+t_{b}\right)\left(P\left(t_{b}\right)-P\left(t_{a}\right)\right)\right] /\left(t_{a}-t_{b}\right)^{3}, \\
& C=t_{b}\left[P^{\prime}\left(t_{a}\right)\left(2 t_{a}^{2}-t_{a} t_{b}-t_{b}^{2}\right)+6 t_{a}\left(P\left(t_{b}\right)-P\left(t_{a}\right)\right)\right] /\left(t_{a}-t_{b}\right)^{3}, \\
& D=-\left[t_{a} t_{b}^{2} P^{\prime}\left(t_{a}\right)\left(t_{a}-t_{b}\right)+t_{b}^{2} P\left(t_{a}\right)\left(t_{b}-3 t_{a}\right)+t_{a}^{2} P\left(t_{b}\right)+\left(3 t_{b}-t_{a}\right)\right] /\left(t_{a}-t_{b}\right)^{3},
\end{aligned}
$$

where the values $P\left(t_{a}\right)$ and $P\left(t_{b}\right)$ are given by the expressions in Eq. (15) evaluated at $t \leq N^{-1}$ or $t \geq 4 N^{-1}$.

\section{Appendix B: Two-particle dispersion wave model}

To derive averaged expressions for the two-particle dispersion resulting from a random superposition of waves, we start from Eq. (17),

$$
\zeta_{i j,(\text { wav })}(t)=z_{i,(\text { wav })}(t)-z_{j,(\text { wav })}(t)=\sum_{\omega} A_{\omega}\left\{\left[\cos \left(\omega t+\phi_{\omega}\right)-\cos \left(\phi_{\omega}\right)\right]-\left[\cos \left(\omega t+\phi_{\omega}^{\prime}\right)-\cos \left(\phi_{\omega}^{\prime}\right)\right]\right\}+\delta z_{0},
$$

where $\phi_{\omega}^{\prime}$ is the phase of the wave with frequency $\omega$ as seen by the particle $j$, which is displaced a distance $\approx \delta r_{0}$ (as $\delta z_{0} \ll \delta r_{0}$ ) from the particle $i$ (with phase $\phi_{\omega}$ ). Thus, $\phi_{\omega}^{\prime} \approx \phi_{\omega}+k \delta r_{0}$ (note we ignore any increase in time of the horizontal distance between the two particles, and in the following we consider only the increase in the vertical distance between them). We can approximate $k \approx K_{\|} / \cos \alpha$, where $\alpha$ is the angle of propagation of the waves with respect to the vertical direction, and $K_{\|}$is the parallel integral wave number as introduced in Sec. II. From the dispersion relation of internal gravity waves we have $\omega=N \sin \alpha$ (or $\sin \alpha=\omega / N)$, and using $\cos \alpha=\left(1-\sin ^{2} \alpha\right)^{1 / 2}$ and that $\omega / N$ in the single-particle model is a random variable uniformly distributed between $1 / 2$ and 1 , we can estimate the mean value of the wavenumber $k$ for an ensemble of waves propagating in all available directions as

$$
\langle k\rangle=\left\langle\frac{K_{\|}}{\cos \alpha}\right\rangle_{\omega / N}=2 K_{\|} \int_{1 / 2}^{1} \frac{d(\omega / N)}{\sqrt{1-(\omega / N)^{2}}} \approx 2.1 K_{\|}
$$

where the factor 2 multiplying the integral comes from computing the mean of $\omega / N$ in the interval $[1 / 2,1]$. Thus, the mean phase shift results to be $\langle\delta \phi\rangle \approx 2.1 K_{\|} \delta r_{0}$.

The two-particle squared vertical dispersion caused by the waves $\left\langle\zeta_{z,(\text { wav })}^{2}\right\rangle$ is the average over an ensemble of particle pairs of the square of the vertical two-particle dispersion for a single pair $\zeta_{i j,(w a v)}$. For a very small initial separation between the particle pairs $\delta \phi$ is also small and we can use in Eq. (B1) the approximation $\cos (\omega t+\phi+\delta \phi) \approx$ $\cos (\omega t+\phi)-\delta \phi \sin (\omega t+\phi)$ to get

$$
\zeta_{i j,(w a v)} \approx \sum_{\omega}-k \delta r_{0} A_{\omega}\left[\sin \left(\omega t+\phi_{\omega}\right)-\sin \left(\phi_{\omega}\right)\right]+\delta z_{0} .
$$

For short times we can take the first order Taylor approximations $\sin (\omega t) \approx-\omega t$ and $\cos (\omega t) \approx 1$. Also, using the trigonometrical identity $\sin \left(\omega t+\phi_{\omega}\right)=\sin (\omega t) \cos \left(\phi_{\omega}\right)+\cos (\omega t) \sin \left(\phi_{\omega}\right)$ we obtain

$$
\zeta_{i j,(w a v)} \approx t \delta r_{0} \sum_{\omega}\left[-\omega k A_{\omega} \cos \left(\phi_{\omega}\right)\right]+\delta z_{0} .
$$

Finally, we take the ensemble average of the square of $\zeta_{i j,(\text { wav })}$, we use that $\alpha$ and $\omega$ are stochastic variables, we use Eq. 13) for $A_{\omega}$, and we use Eqs. (B2), A3) and (A4) to get

$$
\left\langle\zeta_{w a v}^{2}\right\rangle(t) \approx U_{z}^{2}\left(2.1 K_{\|} \delta r_{0}\right)^{2} t^{2}
$$


where terms in $\delta z_{0}$ and $\delta z_{0}^{2}$ are neglected for being much smaller than the leading order term.

To obtain the long time approximation we start by neglecting the term $\delta z_{0}$ and taking the mean squared value of Eq. B1

$$
\begin{aligned}
\left\langle\zeta_{i j,(w a v)}^{2}\right\rangle= & \left\langle\sum _ { \omega } A _ { \omega } ^ { 2 } \left\{\left[\cos \left(\omega t+\phi_{\omega}\right)-\cos \left(\phi_{\omega}\right)\right]^{2}+\left[\cos \left(\omega t+\phi_{\omega}^{\prime}\right)-\cos \left(\phi_{\omega}^{\prime}\right)\right]^{2}-\right.\right. \\
& \left.\left.2\left[\cos \left(\omega t+\phi_{\omega}\right)-\cos \left(\phi_{\omega}\right)\right]\left[\cos \left(\omega t+\phi_{\omega}^{\prime}\right)-\cos \left(\phi_{\omega}^{\prime}\right)\right]\right\}+\sum_{\omega, \omega^{\prime} \neq \omega}(\ldots)\right\rangle,
\end{aligned}
$$

where the mean is taken both over time and over particle pairs. The cross-product terms in Eq. (B6) have mean value

$\left\langle\sum_{\omega, \omega^{\prime} \neq \omega}(\ldots)\right\rangle=0$, as discussed in Appendix A Using again the approximation $\cos \left(\omega t+\phi_{\omega}+\delta \phi\right) \approx \cos \left(\omega t+\phi_{\omega}\right)-$ $\delta \phi \sin (\omega t+\phi)$ we get

$$
\begin{array}{r}
\left\langle\zeta_{i j,(w a v)}^{2}\right\rangle \approx\left\langle\sum _ { \omega } A _ { \omega } ^ { 2 } \left\{\left[\cos \left(\omega t+\phi_{\omega}\right)-\cos \left(\phi_{\omega}\right)\right]^{2}+\left[\cos \left(\omega t+\phi_{\omega}\right)-\cos \left(\phi_{\omega}\right)-\delta \phi\left(\sin \left(\omega t+\phi_{\omega}\right)-\sin (\phi)\right)\right]^{2}-\right.\right. \\
\left.\left.2\left[\cos \left(\omega t+\phi_{\omega}\right)-\cos \left(\phi_{\omega}\right)\right]\left[\cos \left(\omega t+\phi_{\omega}\right)-\cos \left(\phi_{\omega}\right)-\delta \phi\left(\sin \left(\omega t+\phi_{\omega}\right)-\sin (\phi)\right)\right]\right\}\right\rangle .
\end{array}
$$

Finally, using Eqs. A3, A4, A7, A8, and A10 and given that $\langle\sin (\phi) \cos (\phi)\rangle_{\phi}=0$ with $\phi$ uniformly distributed between 0 and $2 \pi$, we have

$$
\left\langle\zeta_{z,(w a v)}^{2}\right\rangle_{t} \approx\left\langle\left(k \delta r_{0}\right)^{2}\right\rangle\left\langle\sum_{\omega} A_{\omega}^{2}[\sin (\omega t+\phi)-\sin (\phi)]\right\rangle_{t} \approx\left\langle\left(k \delta r_{0}\right)^{2}\right\rangle\left\langle\delta z_{w a v}^{2}\right\rangle_{t} \approx \frac{2\left(2.1 K_{\|} \delta r_{0}\right)^{2} U_{z}^{2}}{N \omega_{\min }}
$$

\section{ACKNOWLEDGMENTS}

The authors acknowledge support from PICT Grant No. 2015-3530.

[1] J. C. Wyngaard, "Atmospheric Turbulence," Annu. Rev. Fluid Mech. 24, 205 (1992)

[2] E. A. D'Asaro and R.-C. Lien, "Lagrangian Measurements of Waves and Turbulence in Stratified Flows," J. Phys. Oceanogr. 30, 641 (2000).

[3] T. Watanabe, J.J. Riley, and K. Nagata, "Turbulent entrainment across turbulent-nonturbulent interfaces in stably stratified mixing layers," Phys. Rev. Fluids 2, 104803 (2017).

[4] G. Amir, N. Bar, A. Eidelman, T. Elperin, N. Kleeorin, and I. Rogachevskii, "Turbulent thermal diffusion in strongly stratified turbulence: Theory and experiments," Phys. Rev. Fluids 2, 064605 (2017).

[5] E. Lindborg and G. Brethouwer, "Vertical dispersion by stratified turbulence," J. Fluid Mech. 614, 303 (2008).

[6] M. L. Waite, "Stratified turbulence at the buoyancy scale," Phys. Fluids 23, 066602 (2011).

[7] R. Marino, P. D. Mininni, D. L. Rosenberg, and A. Pouquet, "Large-scale anisotropy in stably stratified rotating flows," Phys. Rev. E 90, 023018 (2014)

[8] A. Maffioli, "Vertical spectra of stratified turbulence at large horizontal scales," Phys. Rev. Fluids 2, 104802 (2017).

[9] L. M. Smith and F. Waleffe, "Generation of slow large scales in forced rotating stratified turbulence," J. Fluid Mech. 451, $145(2002)$

[10] P. Clark di Leoni and P. D. Mininni, "Absorption of waves by large-scale winds in stratified turbulence," Phys. Rev. E 91, 033015 (2015)

[11] N. E. Sujovolsky, P. D. Mininni, and M. P. Rast, "Single-particle dispersion in stably stratified turbulence," Phys. Rev. Fluids 3, 034603 (2018).

[12] Y. Kimura and J. R. Herring, "Diffusion in stably stratified turbulence," J. Fluid Mech. 328, 253 (1996)

[13] Y. Kaneda and T. Ishida, "Suppression of vertical diffusion in strongly stratified turbulence," J. Fluid Mech. 402, 311-327 $(2000)$

[14] L. Liechtenstein, F. S. Godeferd, and C. Cambon, "The role of nonlinearity in turbulent diffusion models for stably stratified and rotating turbulence," Int. J. Heat Fluid. Fl. 27, 644 (2006)

[15] C. Rorai, P. D. Mininni, and A. Pouquet, "Turbulence comes in bursts in stably stratified flows," Phys. Rev. E 89, 043002 $(2014)$

[16] F. Feraco, R. Marino, A. Pumir, L. Primavera, P. D. Mininni, A. Pouquet, and D. Rosenberg, "Vertical drafts and mixing in stratified turbulence: sharp transition with Froude number," EPL in press (2018), arXiv: 1806.00342. 
[17] P. Billant and J.-M. Chomaz, "Self-similarity of strongly stratified inviscid flows," Phys. Fluids 13, 1645 (2001)

[18] S. M. de Bruyn Kops, J. J. Riley, and K. B. Winters, "Reynolds and froude number scaling in stably-stratified flows," Fluid Mech. Appl. 74, 71 (2004).

[19] E. Bauer, "Dispersion of tracers in the atmosphere and ocean: Survey and comparison of experimental data," J. Geophys. Res. 79, 789 (1974).

[20] H. J. S. Fernando, "Turbulent Mixing in Stratified Fluids," Annu. Rev. Fluid Mech. 23, 455 (1991).

[21] K. L. Polzin, J. M. Toole, J. R. Ledwell, and R. W. Schmitt, "Spatial Variability of Turbulent Mixing in the Abyssal Ocean," Science 276, 93 (1997).

[22] C. Wunsch and R. Ferrari, "Vertical Mixing, Energy, and the General Circulation of the Oceans," Annu. Rev. Fluid Mech. 36, 281 (2004).

[23] G.N. Ivey, K.B. Winters, and J.R. Koseff, "Density Stratification, Turbulence, but How Much Mixing?" Annu. Rev. Fluid Mech. 40, 169-184 (2008).

[24] P. Klein and G. Lapeyre, "The Oceanic Vertical Pump Induced by Mesoscale and Submesoscale Turbulence," Annu. Rev. Mar. Sci. 1, 351 (2009)

[25] L.A. Mingari, E.A. Collini, A. Folch, W. Báez, E. Bustos, M.S. Osores, F. Reckziegel, P. Alexander, and J.G. Viramonte, "Numerical simulations of windblown dust over complex terrain: the Fiambalá Basin episode in June 2015," Atmos. Chem. Phys. 17, 6759 (2017).

[26] A. Jones, D. Thomson, M. Hort, and B. Devenish, "The UK Met Office's next-generation atmospheric dispersion model, NAME III," in Air pollution modeling and its application XVII (Springer, 2007) pp. 580-589.

[27] F. S. Godeferd, N. A. Malik, C. Cambon, and F. Nicolleau, "Eulerian and Lagrangian statistics in homogeneous stratified flows," Appl- Sci. Res. 57, 319 (1996).

[28] F. Nicolleau and J. C. Vassilicos, "Turbulent diffusion in stably stratified non-decaying turbulence," J. Fluid Mech. 410, $123(2000)$

[29] M. van Aartrijk, H. J. H. Clercx, and K. B. Winters, "Single-particle, particle-pair, and multiparticle dispersion of fluid particles in forced stably stratified turbulence," Phys. Fluids (1994-present) 20, 025104 (2008)

[30] J. Bec, L. Biferale, M. Cencini, A. Lanotte, S. Musacchio, and F. Toschi, "Heavy Particle Concentration in Turbulence at Dissipative and Inertial Scales," Phys. Rev. Lett. 98, 084502 (2007).

[31] S. Sumbekova, A. Cartellier, A. Aliseda, and M. Bourgoin, "Preferential concentration of inertial sub-kolmogorov particles: The roles of mass loading of particles, stokes numbers, and reynolds numbers," Phys. Rev. Fluids 2, 024302 (2017).

[32] M. van Aartrijk and H. J. H. Clercx, "Vertical dispersion of light inertial particles in stably stratified turbulence: The influence of the Basset force," Phys. Fluids 22, 013301 (2010).

[33] A. Sozza, F. De Lillo, S. Musacchio, and G. Boffetta, "Large-scale confinement and small-scale clustering of floating particles in stratified turbulence," Phys. Rev. Fluids 1, 052401(R) (2016)

[34] M. P. Rast and J.-F. Pinton, "Pair Dispersion in Turbulence: The Subdominant Role of Scaling," Phys. Rev. Lett. 107, $214501(2011)$

[35] M.P. Rast, J.-F. Pinton, and P.D. Mininni, "Turbulent transport with intermittency: Expectation of a scalar concentration," Phys. Rev. E 93, 043120 (2016).

[36] P. D. Mininni, D. Rosenberg, R. Reddy, and A. Pouquet, "A hybrid MPIOpenMP scheme for scalable parallel pseudospectral computations for fluid turbulence," Parallel Comput. 37, 316 (2011)

[37] P.K. Yeung and S.B. Pope, "An algorithm for tracking fluid particles in numerical simulations of homogeneous turbulence," J. Comp. Phys. 79, 373 (1988).

[38] N. E. Sujovolsky, P. D. Mininni, and A. Pouquet, "Generation of turbulence through frontogenesis in sheared stratified flows," Phys. Fluids 30, 086601 (2018).

[39] P. Billant and J.-M. Chomaz, "Theoretical analysis of the zigzag instability of a vertical columnar vortex pair in a strongly stratified fluid," J. Fluid Mech. 419, 29 (2000).

[40] J.J. Riley and S.M. deBruynKops, "Dynamics of turbulence strongly influenced by buoyancy," Phys. Fluids 15, 2047 (2003).

[41] P. A. Davidson, Turbulence in Rotating, Stratified and Electrically Conducting Fluids (Cambridge University Press, 2013). 